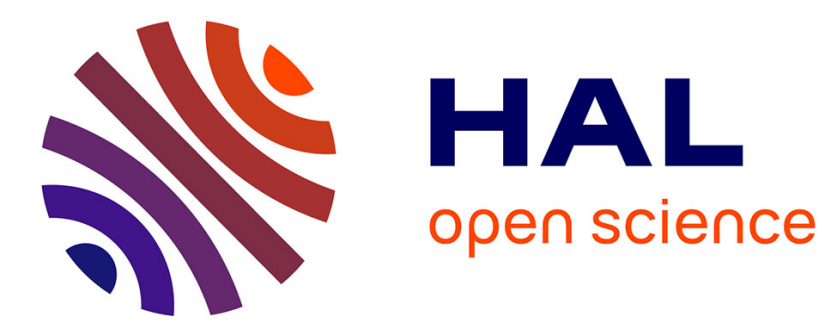

\title{
L'exploration archéologique de Bavai
}

Henri Biévelet

\section{To cite this version:}

Henri Biévelet. L'exploration archéologique de Bavai. Gallia - Fouilles et monuments archéologiques en France métropolitaine, 1943, 1 (2), pp.159-189. 10.3406/galia.1943.1973 . hal-01920078

\section{HAL Id: hal-01920078 https://hal.science/hal-01920078}

Submitted on 25 Feb 2020

HAL is a multi-disciplinary open access archive for the deposit and dissemination of scientific research documents, whether they are published or not. The documents may come from teaching and research institutions in France or abroad, or from public or private research centers.
L'archive ouverte pluridisciplinaire HAL, est destinée au dépôt et à la diffusion de documents scientifiques de niveau recherche, publiés ou non, émanant des établissements d'enseignement et de recherche français ou étrangers, des laboratoires publics ou privés.

\section{(이)(\$)}

Distributed under a Creative Commons Attribution - NonCommercial - NoDerivatives| 4.0 


\title{
L'EXPLORATION ARCHEOLOGIQUE DE BAVAI
}

\author{
par M. l'abbé Henri BiÉvelet
}

César, au livre II du De Bello Gallico, raconte longuement la bataille où les Nerviens faillirent le vaincre en 57 avant Jésus-Christ; il nomme le chef qui les commandait, Boduognatus, et la rivière sur les bords de laquelle il fut aux prises avec eux, Sabis: Au livre V, il s'étend sur les dangers que, trois ans plus tard, ils firent courir, avec Ambiorix, les Éburons et les Aduatiques, à $Q$. Cicero et à sa légion; il nous transmet le nom du seul Nervien qui, en cette occasion, ait voulu partager le sort des Romains, Vertico. Mais il néglige de noter quelle était la capitale de cette nation valeureuse (1).

Il faut attendre la première moitié $d u{ }_{I^{e}}{ }^{e}$ siècle pour trouver mention de Bavai. Ptolémée, après avoir parlé des Tongres, écrit : "Au-dessous de ces peuples s'étendent, les plus au Nord, les Nerviens, dont la cité est Bavai (2)."

Les mots Bagaco Nervio[rum] sont inscrits sur la Table de Peutinger, accompagnés des deux tourelles à toit pointu qui, dans ce document, désignent, sauf exception, les villes capitales (3). On sait que cette carte date, pour le fond, de la première moitié du III ${ }^{\mathrm{e}}$ siècle. Vers la fin du même siècle, l'Itinéraire d'Antonin nomme trois fois Bagacum et une fois Bagacum Nerviorum (4).

On le voit : les textes anciens sont peu nombreux à nous parler de Bavai et ils le font fort brièvement.

Mais, en dépit des injures du temps et malgré les coups que trop souvent leur ont portés les invasions et les guerres, les édifices construits dans cette ville par les bâtisseurs romains n'ont pas complètement disparu; vers elle

(1) De Bello Gallico, II, 23, 4 : « ... Boduognato, qui summam imperii tenebat... »; II, 16, 1 : « Sabim flumen... *; V, 45, 2 : "... Nervius, nomine Vertico... ".

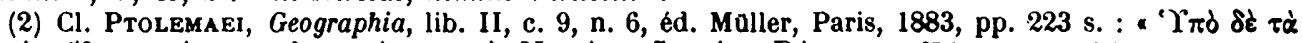

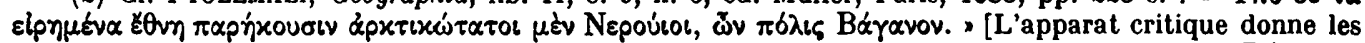

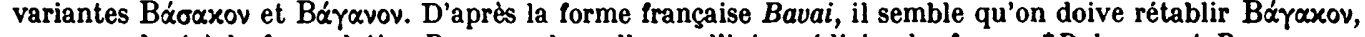
correspondant à la forme latine Bagacum, laquelle, par l'intermédiaire des formes *Babacum et Bavacum, a abouti phonétiquement à Bavai. (Note de M. René Lours, professeur à la Faculté des Lettres de Lille, directeur de la Ire circonscription archéologique historique.)]

(3) Cl. Rev. Et. Anc., XIV, 1 (1912), pl. III.

(4) Otto Cunrz, Teubner, 1929, Itineraria Romana, vol. I, Itineraria Antonini Augusti et Burdigalense, p. 58 , n. 376,$2 ;$ n. 377,$1 ;$ n. 378,1 . 
montent encore de tous les coins de l'horizon, pour y former un remarquable carrefour, les routes que suivaient les légions; le sol où elle se survit n'a point cessé d'être un champ de fouilles particulièrement fertile.

A considérer quelle attention fut prêtée jusqu'à présent à ces divers monuments du passé, on comprendra mieux l'importance comme l'opportunité des mesures qui sont intervenues le 4 juillet 1942 en faveur de l'ancienne capitale de la Nervie.

\section{I. - Du Moyen Age à la fin du XVII e siècle.}

"Chastel " ou " castelet " est le nom sous lequel étaient connues, au Moyen tge, les ruines imposantes qui étaient groupées au N.-O. de la ville et qui sont en bonne partie restées visibles (1).

Au XIve siècle, Lucius de Tongres voit dans ces ruines celles d'un palais que, s'il fallait l'en croire, aurait élevé, en fondant Bavai, Bavo, le cousin de Priam. La description qu'il nous en donne et que nous a gardée Jacques de Guyse, n'est pas pure fantaisie. Il écrit par exemple : "Toutes ces constructions formaient des masses bien solides, sans vides, constituces par un béton fait de briques broyées et mêlées aver de la chaux... Ce palais, quant à la forme, était long et étroit à la façon d'un navire. La partie principale du palais était pavée de pierres d'un grand prix, comme le constatent ceux qui creusent là un peu profondément $(2)$."

De sa ville natale Jean Lemaire de Belges écrit, en 1512, dans les Illustralions de la Gaule et Singularitez de Troye : "Bauais en Haynau... à présent n'est qu'une petite ville déserte et désemparée ; mais les ruines dicelle monstrent bien que au temps passé elle ha esté de merveilleuse estendue (3). ”

Dans ses Rerum Belgicarum Annales, dont la dédicace est datée de 1624, Aubert Lemire est le premier témoin, à notre connaissance, d'une tradition d'après laquelle les ruines du N.-O. de la ville seraient les restes d'un cirque romain : "On va voir aujourd'hui encore, dans cette ville, les murs en ruines d'un cirque bâti par les Romains; il est de forme oblongue, à la façon d'un navire ; on l'appelle le "Vieu-Chastel " (4).

(1) C1. au plan la rue du Chastelaire.

(2) Jacques DE GUYSE, Annales historiæ illustrium principum Hannoniæ, lib. I, cap. XIII, édit. Fortia d'Urban, Paris et Bruxelles, 1826, t. II, p. 235 : "Omnia siquidem dicta ædificia... erant admodum solidæ massæ non concavæ, de bitumine compacto ex lateribus pulverisatis cum calce artificialiter permixtis... Hoc autem palatium... quantum ad formam longum et strictum ad modum navis protendebalur... Erat autem planities intrinseca palatii principalioris partis lapidibus preciosis constrata, prout patet ibidem terram aliquantulum profunde effodientibus. $\mathrm{Si}$ on $\mathrm{n}$ 'a pas trahi la pensée de Lucius de Tongres en traduisant bitumen par béton, tous les traits de cette description relevés ici sont exacts.

(3) Ed. Stecher, Louvain, 1882, t. II, p. 290.

(4) P. 39 : In eodem oppido hodieque visuntur muri ac ruinæ circi a Romanis structi, forma oblonga, in modum navis; vulgo "Vieu-Chastel "vocant..." 


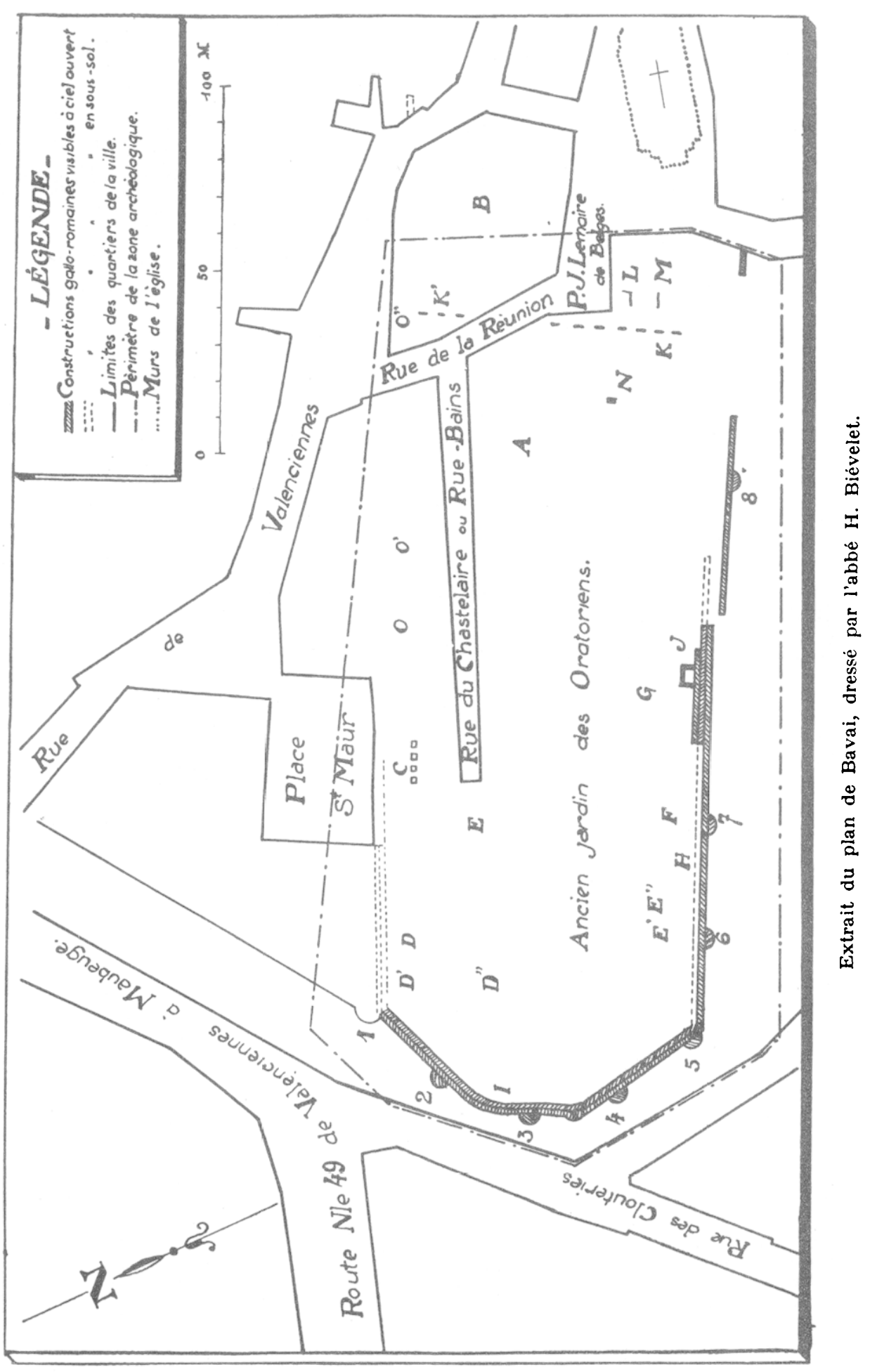


Aubert Lemire nous signale d'autres ensembles de ruines romaines qui étaient visibles jadis et qui ont cessé de l'être ou furent détruites : "... sous l'église paroissiale de Bavai les murs dégradés d'une sorte de crypte subsistent encore de nos jours ; les savants conjecturent que ce sont des restes de thermes... Hors de la ville de Bavai on va voir un aqueduc remarquable; partant de la ville même, du côté de Hautmont, il s'étend à plus de trois milles au delà de la Sambre, jusqu'à Floursies... (1) ". "Sur les cloaques, jusqu'à présent je n'ai rien trouvé. Je sais ceci : dans la campagne voisine aujourd'hui encore des cryptes ou des galeries souterraines font l'admiration de ceux qui les voient; mais quelle en était anciennement la destination, je ne le sais pas (2). "

(1) Ouv. cit., p. 40 : "Sub templo parochiali Bavacensi, parietinæ velut cryptæ etiamnum resiant, quæ thermarum reliquias esse eruditi suspicantur... Extra oppidum Bavacense visitur aquæductus insignis, qui facto initio ab ipso oppido, versus Altum Montem... ullra Sabim, usque Fontem Floridum... ad tria amplius miliaria extenditur. "

(2) P. 42 : "De cloacis nihil mihi adhuc compertum; hoc scio, in agro suburbano hodieque cryptas seu vias sublerraneas cum admiratione spectari, sed in quem olim usum nescio. »

S'il fallait en croire une chanson de geste qui date de la première moitié du xive siècle, Li Romans de Bauduin de Sebourc, èd. B. Henry, Valenciennes, 1841, XIX, v. 705 à v. 725 et XX, v. 297 à v. 305 et v. 316 à v. 320, Bavai, Sebourg et Famars auraient été reliés par un souterrain établi par les " payens ». C'est ainsi qu'aux vers 708 et suivants du chant XIX, on lit :

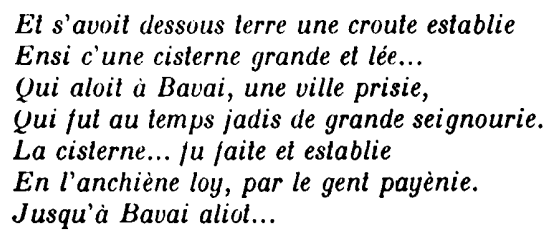

Dans son Etude sur Bauduin de Sebourc, Paris, 1940, p. 68, M. Edmond-René Labande écrit de l'auteur de ce poème : * Il connaissait... (la région) qui se trouve au sud et au sud-est de (Valenciennes). En effet, on voit aux vers XIX, 710 et 716, les villages de Famars et de Bavai reliés au château de Sebourc par des souterrains ; non seulement ce n'est pas une invraisemblance, étant donné le peu de distance entre les points considérés, mais c'est un élément facile à contrôler. Il existe encore de nos jours d'immenses galeries souterraines dans les environs de Bavai, portant le nom de *Trous des Sarrasins ": ce sont probablement des carrières de calcaire largement exploitées à l'époque romaine. „Et $M$. Labande renvoie ses lecteurs à l'étude de $M$. Desailly, publiée dans le Bulletin de la Société préhistorique française, t. XX [1923], pp. 3u6 ss. et intitulée : Notice sur les souterrains connus sous le nom de trous des Sarrasins des environs de Bavai (Nord) .

A vol d'oiseau, entre la ville de Bavai et le village de Famars il y a 20 kilomètres. Le peu de distance qui sépare deux points ne sumt pas à rendre vraisemblable l'affirmation qu'un souterrain les relie. Il n'est d'ailleurs pas facile de s'assurer qu'il existe dans les environs de Bavai d'immenses galeries souterraines. L'entrée du trou des Sarrasins, situé sur le territoire de Houdain-lez-Bavai, le seul qui fût encore accessible avant 1940, est pour le moment obstruée; ce qu'on pouvait voir de ce souterrain était de proportions modestes.

Aussi bien vaut-il mieux sans doute ne pas chercher à quelques kilomètres de Bavai et de la route de Bavai à sebourg une explication qu'on peut trouver à Bavai même. Il y a des souterrains dans lè sous-sol de cette localité. Depuis Lemire, plusieurs auteurs en ont fait mention, sans donner beaucoup plus de précisions que lui. Cif, par exemple, Lebeau, Archives historiques et littéraires du Nord de la France et du Midi de la Belgique, nouvelle série, V, p. 143, et Delhaye, Bavai et la contrée quí l'environne, Douai, 1869, pp. 283 ss. On peut croire qu'au xiv ${ }^{e}$ siècle, comme de nos jours, le public en connaissait l'existence, sans bien savoir queltes pouvaient en être l'exacte nature et les dimensions, ce qui mettait le poète à l'aise pour en parler comme il le fit.

Il faut noter que la tradition est gardée jusqu'à présent à Bavai d'un souterrain entre cette ville et Famars. 
A parler de tels monuments Aubert Lemire s'est ému et il termine la page qu'il consacre à Bavai par une apostrophe toute lyrique : "Bavai, toi qui fus jadis la Rome de la Belgique, ... relève donc un peu la tête de tes ruines, si tu le peux! (1)"

Par son Rerum Belgicarum Chronicon, qui date de 1636, nous savons qu'Aubert Lemire est venu visiter Bavai en 1633 et que lors de son passage on mettait au jour, près de la porte de Valenciennes, d'importantes substructions : "... des fondations de thermes ou d'un très vaste palais, des marbres et des pierres de grandes dimensions étaient découverts à Bavai, dans la campagne prochaine, près de la porte de Valenciennes, en 1633, quand je suis allé voir les antiquités romaines de cette localité (2)."

Il est possible que ce soit les mêmes substructions que visent les Bollandistes, dans les Acta Sanctorum, en 1643, quand ils parlent de Bavai, à propos des reliques de saint Maur qu'on y avait vénérées : «... les fondations de grands édifices sont découvertes çà et là dans l'enceinte de cette ville, comme nous-mêmes, nous l'avons constaté en 1630 et 1633 (3). " Alors que pour Aubert Lemire Bavai avait été la Rome de la Belgique, les Bollandistes nous gardent le témoignage d'une opinion plus réservée : "Bavai est une ville très ancienne du Hainaut et, comme le pensent des savants, le principal poste romain en Nervie (4)."

Gilles Boucher, lui aussi, a visité Bavai. Fort de ses constatations, il corrige, dans son Belgium Romanum Ecclesiasticum el civile, de 1655, une affirmation, non de Jacques de Guyse, comme il le dit, mais de Lucius de Tongres, affirmation reprise par Aubert Lemire, sur les ruines du prétendu cirque : " Jacques de Guyse écrit que ce cirque a la forme d'un navire... Cependant je crois avoir bien remarqué que les murs en sont, non courbes et ovales, mais droits et presque parallèles. Aussi, à mon sens, est-il presque semblable au Cirque Maxime de Rome, bien que plus petit... Mais les habitants n'y reconnaissent pas un cirque, non plus que Jacques de Guyse ; pour celui-ci c'est un palais ; pour eux, un château ; on l'appelle le Chastelet (5). "

Les ruines d'un aqueduc ont tout particulièrement frappé Boucher : "Rien

(1) Ouv. cit., pp. 42 ss. : "Bavacum, Belgica olim Roma, ... paulisper e ruinis tuis, si fas est, faciem altole. *

(2) Autberti Miræi rerum Belgicarum Chronicon, Anvers, 1636, p. 129 : "Thermarum aut certe palatii fundamenta, marmora et saxa prægrandia in agro suburbano ad portam Valentinianensem, Bavaci, anno 1633, eruebanlur, cum ad lustrandas illius loci antiquitates Romanas excurrissem. "

(3) Acta Sanctorum, Anvers, 1643, t. I, p. 1051 : "Ingentium ædificiorum fundamenta ... passim in pomœrio eruuntur, ut ipsi anno 1630 et 1633 coram adspeximus."

(4) Ibid. : "Est Bavacum vetustissimum Hannoniæ oppidum et, ut quidam eruditi censent, præcipua Romanorum in Nerviis statio."

(5) R. P. Egidii Bucherii Atrebatis... Belgium Romanum ecclesiasticum et civile, liv. XVI, cap. VII, 3, p. 502 : a... Guisius... navis illum (circum) figuram expressisse scribit... Ego tamen laterales ejus muros non inflexos et ovales sed reclos et fere parallelos advertisse videor. Unde circo Romæ Maximo fere similem licet minorem existimavi... Sed circum non agnoscunt inquilini neque Guisius; palatium hic, illi castellum, vulgo "Chastelet "vocant. 
ne provoque autant l'admiration du spectateur que la vue d'un très grand aqueduc, qui jusqu'à présent est assez bien conservé en de nombreux endroits... C'est un ouvrage tout à fait admirable, vraiment digne des Romains, que nous avons examiné plusieurs fois et longuement avec une grande satisfaction et non moins d'admiration (1). "

Le carrefour où s'élève Bavai (2) retenait aussi l'attention des curieux, ce carrefour où se rencontrent notamment la voie qui vient de Boulogne et celle que Camille Jullian appelle " la route vitale de l'Europe du Nord », la route " qui va de Cologne à Paris et qui longe la Meuse et la Sambre pour gravir ensuite le seuil du Vermandois et redescendre vers l'Oise ou vers la Somme " (3).

"Les mers feront la fin des sept chaussées Brunehault ", lisait-on sur l'aiguille hentagonale qui, au xvirre siècle, se dressait au milieu de la place de Bavai. Au jugement de Camille Jullian, "la tradition des sept chemins de Brunehault à Bavai remonte au moins au xıIre siècle " (4). Qu'ils soient attribués à Bavo, ou à l' " archidruide "Brunehaldus, ou à la reine d'Austrasie, Brunehault, peu importe : ces fantaisies se valent; mais de telles attributions témoignent qu'on reconnaissait aux sept chaussées une haute antiquité.

$\mathrm{Au}$ bord des chaussées, auprès des ruines, sur tout le territoire qui entoure l'agglomération bavaisienne, on peut penser que les trouvailles de sépultures, d'objets anciens de tout genre et de toute nature furent au moins aussi fréquentes dans le passé qu'à présent. Ces lignes d'Aubert Lemire en sont une preuve pour la première moitié du Xvıre siècle : " Dans la ville de Bavai et les environs, écrit-il, un très grand nombre de monnaies, des poteries et d'autres monuments de l'antiquité sont découverts tous les jours (5). "Il nous parle d'une collection de médailles recueillies à Bavai : "De notre temps, Charles de Crö̈, duc d'Arschot, célébré par Juste Lipse comme le Lucullus de la Belgique, a possédé un remarquable ensemble de monnaies trouvées à Bavai (6). "

(1) Ibid. : . Nihil æque spectatorem in admirationem rapit quam immanis aquæductus multis adhuc locis satis integer... opus sane admirabile, planeque Romanum, quod nos aliquoties diutiusque, magno animi voluptate nec minore admiratione consideravimus. *

(2) ... Bavai, où gist mainte chaucie, lit-on dans Li Romans de Baudoin de Sebourc, XX, 302.

(3) Histoire de la Gaule, t. II, p. 472. Sur la route Boulogne-Bavai-le Rhin, cf. Franz Cumonr, Comment la Belgique fut romanisée, dans Annales de la société royale d'archéologie de Bruxelles, t. XXVIII, 1914, pp. $88 \mathrm{sq.}$ Il est remarquable que les seuls textes qui nous parlent de Bavacum soient trois des cinq «documents itinéraires de l'antiquité " (cf. A. Grenier, Manuel d'archéologie gallo-romaine, II' Partie, « Les routes », p. 126).

(4) Ouv. cit., t. V, p. 102, n. 2. "Les Nerviens, écrit par ailleurs Camille Jullian, ont de tout temps attiré les curieux et les érudits, surtout à cause des fameuses routes rectilignes qui partaient de Bavai et auxquelles la tradition donna le nom de chaussées Brunehault. Cette tradition ne m'a point paru antérieure à l'an 1000, car je ne trouve pas mention de Brunehaut, reine des Francs, avant un texte de l'Historia Francorum d'Aimoin " (Bulletin archéologique du Comité des Travaux historiques et scientifiques, 1926, p. $\mathrm{CXXI}$ ).

(5) Rerum Belgicarum Annales, p. 41 : a In oppido et agro Bavacensi, plurima quotidie numismata, opera item figulina, aliaque antiquitatis monumenta eruuntur. "

(6) Ibid. : Ex numismatibus ibi repertis insignem nostra memoria thesaurum ac copiam habuit Carolus Croylus, dux Arschotanus, ut Lucullus Belgicus a Lipsio celebratus. " 
Dès 1570, dans son Theatrum Orbis Terrarum, Abraham Ortell, énumérant les principales villes du Hainaut, écrivait : "Il y a ... Bauais, laquelle aucuns estiment être ce que Ptolémée a appelé Baganum ou Bavacum. Autres cuident que c'est ce que César appelle en ses commentaires Belgium. Hubertus Leodius ne croit point que du temps de Jules César ceste ville ayt esté si puissante; mais que plustot elle ayt esté fleurissante du temps de l'empereur Constantin ; ce qu'il recueille de ce que journellement on y trouve des médailles, esquelles se voit la figure du dit empereur (1)."

\section{II. - Pendant le $X V I I I^{\mathrm{e}}$ et le $X I X^{\mathrm{e}}$ siècle.}

Des études que le $\mathrm{xvin}^{\mathrm{e}}$ et le $\mathrm{xIx}^{\mathbf{e}}$ siècles nous ont laissées sur Bavai et les monuments de son passé, certaines se distinguent par la précision et l'exactitude des renseignements qu'elles nous donnent.

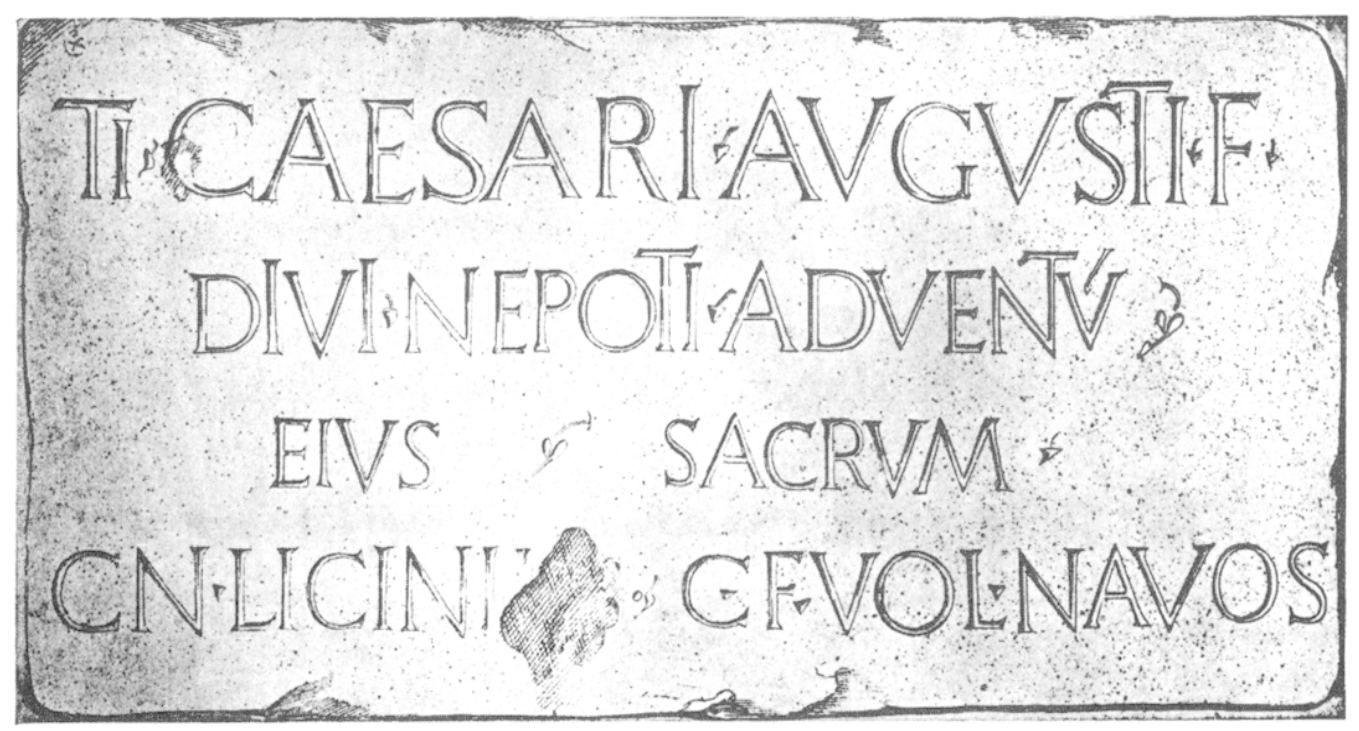

Fig. 1. L'inscription de Tibère, en pierre calcaire (longueur : $1 \mathrm{~m} .40$; largeur : $0 \mathrm{~m}$. 72) trouvée dans le jardin des Oratoriens ( $A$ du plan), aujourd'hui au Musée de Douai (Corpus Inscriptionum latinarum, XIII, 3.570).

Telle d'abord celle de Claude Masse. Cet ingénieur militaire avait reçu mission en 1724 de dresser la carte de la frontière des Pays-Bas et de l'Allemagne. Avec la carte de la région de Bavai il leva le plan de la ville auquel il joignit

(1) On a cité l'édition française, Théatre de l'Univers contenant les cartes de tout le monde, avec une breve déclaration d'icelles, par Abraham Ortelius, le tout reveu par le mesme autheur, Anvers, 1598. 
un mémoire daté du 30 mars 1732 (1). Le mémoire est mal écrit, mal composé ; mais Masse y a consigné ainsi que sur le plan de précieuses observations.

Parmi d'autres découvertes alors récentes, il signale la belle inscription dite de Tibère, trouvée en 1716 et qui est, comme le constatait Camille Jullian, "l'un des très rares monuments de la Gaule mentionnant un fait historique " (2)

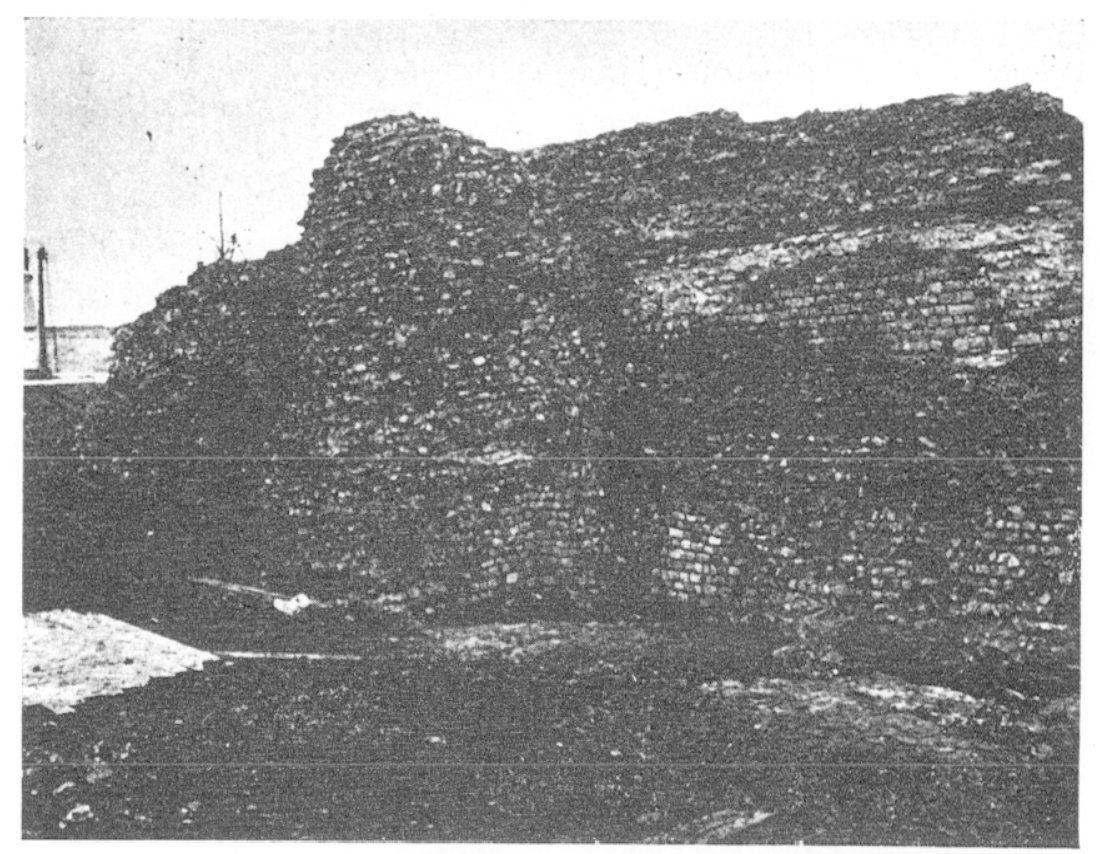

Fig. 2. La courtine ouest et la tour 4 de l'enceinte. (Cf. le plan.)

(fig. 1). Elle nous conserve le souvenir d'un passage du futur empereur à Bavai (3). "... Les restes des aqueducs de Neuf-Mesnil, de Louvignies jusqu'à Bavai, écrit Masse, les vestiges des bains que les ouvriers m'ont assurez d'avoir détruits dans la jardin et prairie des prêtres de l'Oratoire..., les masures des murs du Vieu-Château... et le nombre infini de médailles des empereurs romains, les unes d'or, les autres d'argent, d'autres de cuivre et de bronze, avec des inscriptions latines et grecques... font une preuve incontestable de l'ancienneté de Bavai... les chaussées militaires... sont des preuves de l'importance de cette ville (4). »

(1) Ces documents, qui sont conservés à la bibliothèque du Comité technique du Génie sous le n 191 , ne devaient être publiés qu'en 1912, par Lucien Lemaire, dans les Mémoires de la Société archéologique d'Avesnes, t. X, pp. 79 ss.

(2) Ouv. cit., t. VI, p. 467 , n. 3.

(3) Nous savons par C. Velleius Paterculus que Tibère monta vers la Germanie en 7, 8 et 10 (II, 104 ; 106-107; 120 sq.).

(4) Ouv. cit., p. 103. 
"Outre ce que lon voit visiblement les habitans et ouvricrs qui ont été dans les souterrains qui sont sous l'église, sous la place et aillcurs sont des marques de son antiquité, mais lon en a bouché les entrées pour éviter les désordres et dégradations, ils disent qu'il y a de belles chambres bien pavées et les murs peints (sic) (1). ")

Au N. O. de la ville, Masse, le premier, reconnait les restes d'une enceinte fortifiée de l'époque gallo-romaine (fig. 2 et 3 ). Il en note les matériaux, en étudie la construction qu'il juge "singulière " (2).

Claude Masse avait séjourné à Bavai et il fait état dans son mémoire de ses propres enquêtes. Le comte de Caylus, lui, travaille dans son "cabinet ", et, au tome second de son Recueil d'Antiquités Egyptiennes, Étrusques, Grecques et Romaines publié à Paris en 1756 , il nous parle de Bavai à propos d'objets (fig. 4 et 5) et à l'aide de rapports qu'il en a reçus. Après avoir donné une " ... idée générale de la position et de l'ancienne

(1) Ibid., p. 113.

(2) Ibid., p. 118.

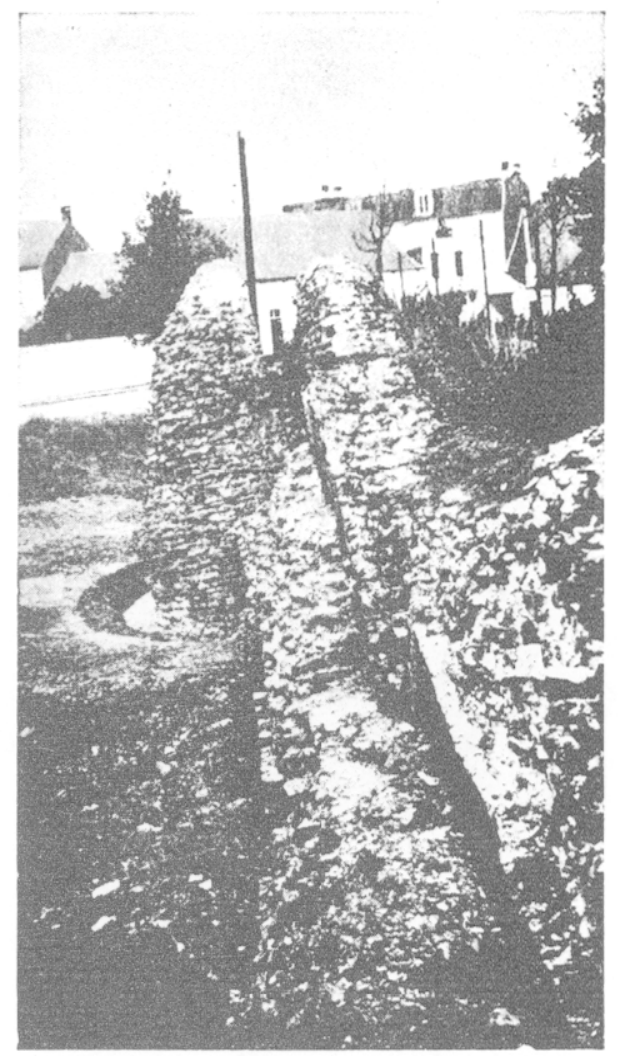

Fig.3. La courtine 0 . et la tour 4 vues de la tour 5.

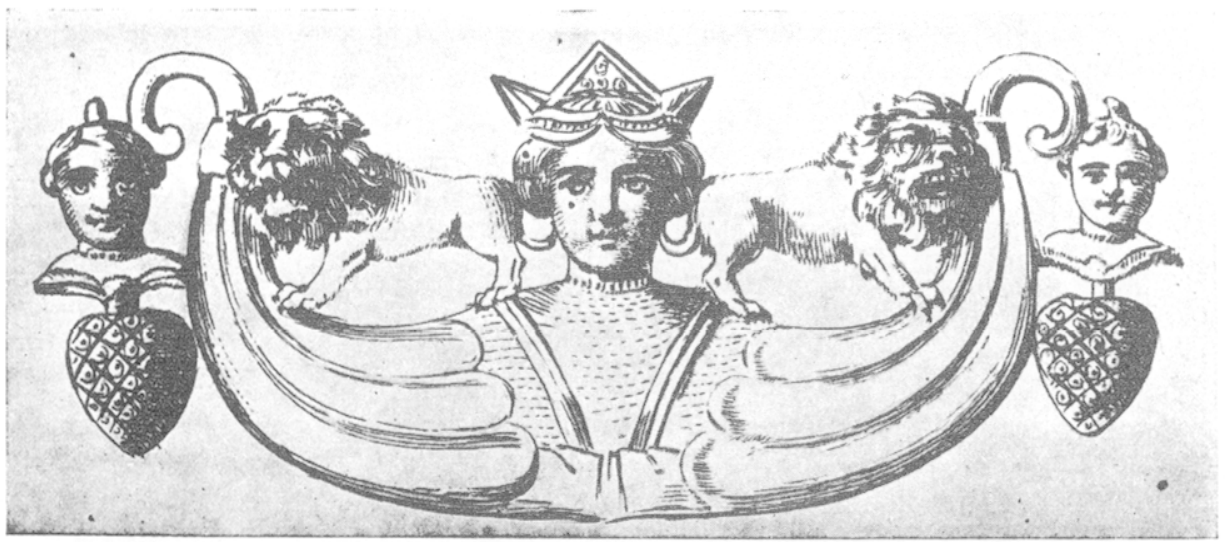

Fig. 4. Poignée de bronze avec motifs empruntès à l'iconographie métroaque. (D'après Caylus, ouv. cit., p. 398, pl. CXVIII, n 6.) Cf. Appendice, no 1, p. 188. 
splendeur de cette ville... "(1), il étudie vingt-quatre pièces diverses, vingt fragments de poterie sigillée et une mosaïque qui avaient été trouvés à Bavai et que reproduisent huit planches bien gravées (2) (fig. 6).

Né à Bavai, en 1741, le récollet Jean-Baptiste Lambiez y était revenu à la Révolution. Dans les ouvrages abondants où il parle de sa ville natale, il fait surtout preuve d'imagination. Mais c'est à lui que revient le mérite d'avoir procédé aux premières fouilles intéressantes

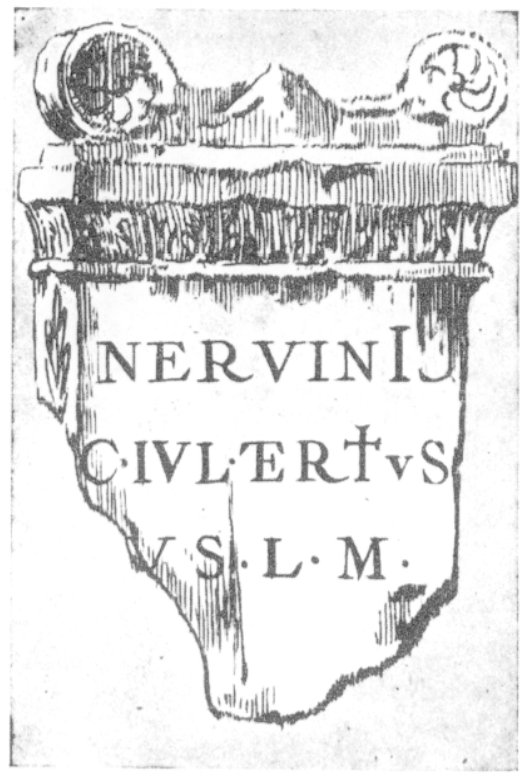

Fıg. 5. Cippe de la collection Caylus (aujourd'hui au Cabinet des Médailles). (Corpus Insc. lat., XIII, 3.569.) Cf. Appendice, $n^{\circ} 2$, p. 188. qui nous soient connues. Pour leur assurer les ressources nécessaires, une souscription avait été autorisée ; un périodique en publia les résultats : c'était la Feuille d'or ou annonce des excavations et des curieuses découvertes... dans l'étendue du département du Nord. Les travaux commencèrent le $1^{\text {er }}$ octobre 1790. Des recherches furent effectuées au N. O. de la ville et des puits furent curés. Il semble bien que dès la fin de novembre les fouilles avaient cessé ; elles ne laissaient pas d'avoir été productives. Notamment on avait trouvé, dans un puits, au bord de la chaussée d'Utrecht, le trépied dit de Bacchus (fig. 7 et 8). Au livre IX de son Histoire monumentaire du Nord des Gaules, appuyée sur les traces marquantes et les vestiges durables des anciennes colonies qui ont illustré les fastes Belgiques, où il traite, avec une belle assurance, de " la fondation de Bavai, la Bacchopole (1176 avant l'ère vulgaire) ", Lambiez reproduit cette pièce, qui est fort belle, et la décrit (3).

Augustin Carlier, originaire, lui, de Boulogne-sur-Helpe, après avoir été vicaire à Bavai, pendant dix-sept ans, y devint curé en 1775 et le resta quarante-trois ans. Il eut tout le temps de se constituer un cabinet d'antiques qui formait un vrai musée. On y pouvait voir plus de deux cents monnaies gauloises, dont dix en or, près de cinq mille monnaies romaines, des vases de

(1) Ouv. cit., p. 394.

(2) Ouv. cit., pl. CXVIII à CXXV. Il y a lieu de compter aussi l'animal accroupi que représente le cul-de-lampe de la p. vir. " Ce petit morceau, dit de Caylus, a été trouvé l'année dernière à Bavai d'où il m'a été envoyé - (p. v). Semblable objet, en poterie blanche, a été trouvé près de la chaussée d'Utrecht, en mai 1940.

(3) Histoire monumentaire du Nord des Gaules..., t. I, Mons, pp. 231 ss. 


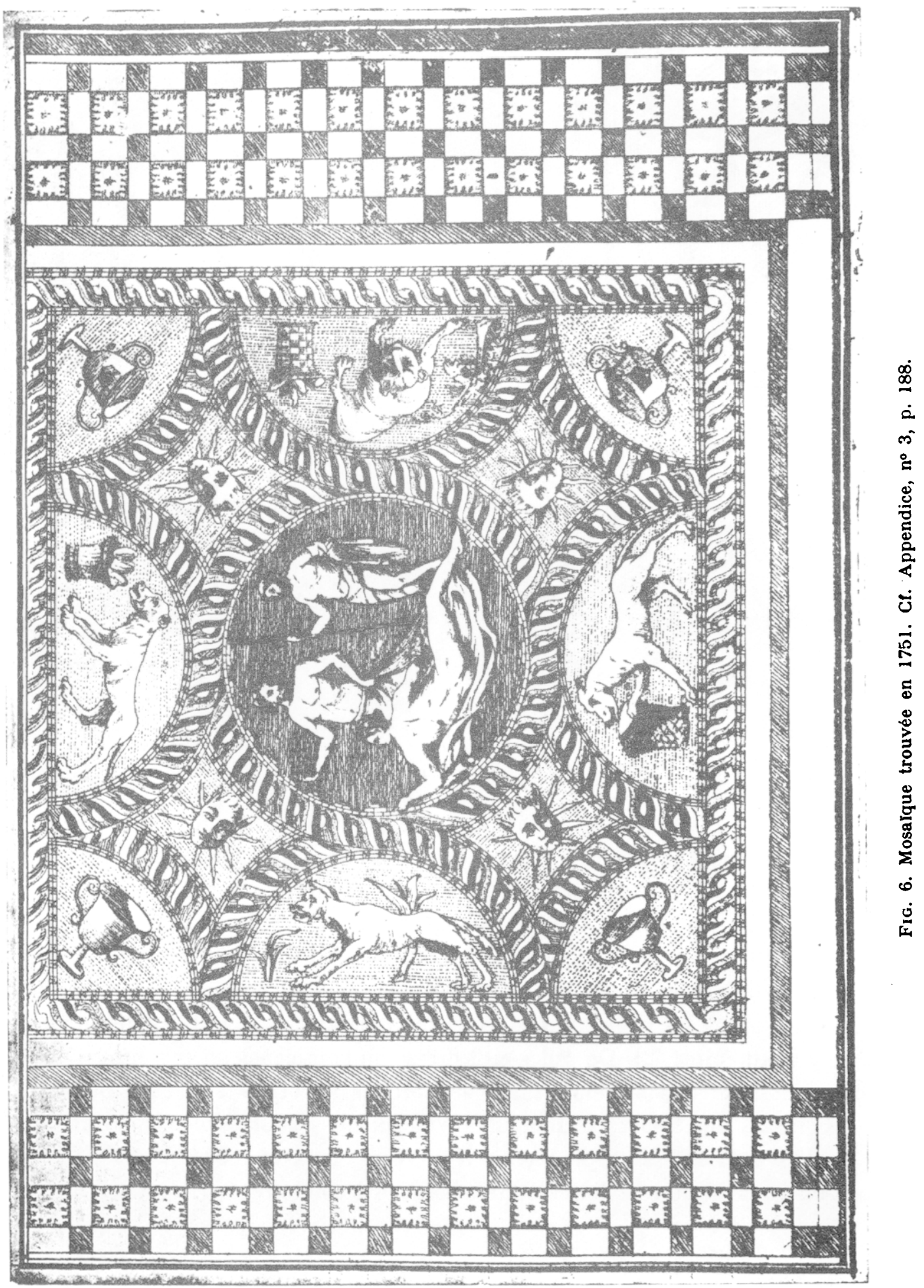




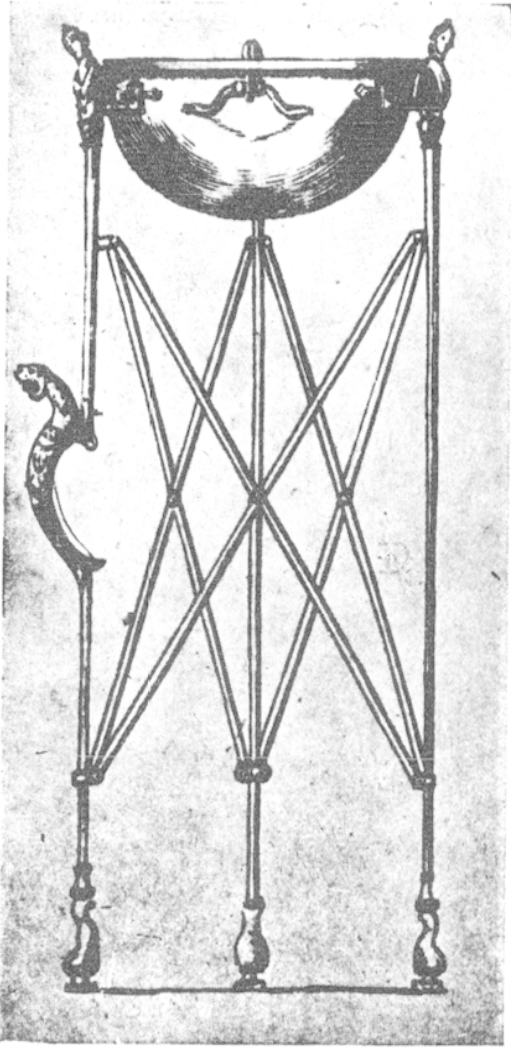

Fig. 7. Le trépied de Bacchus.

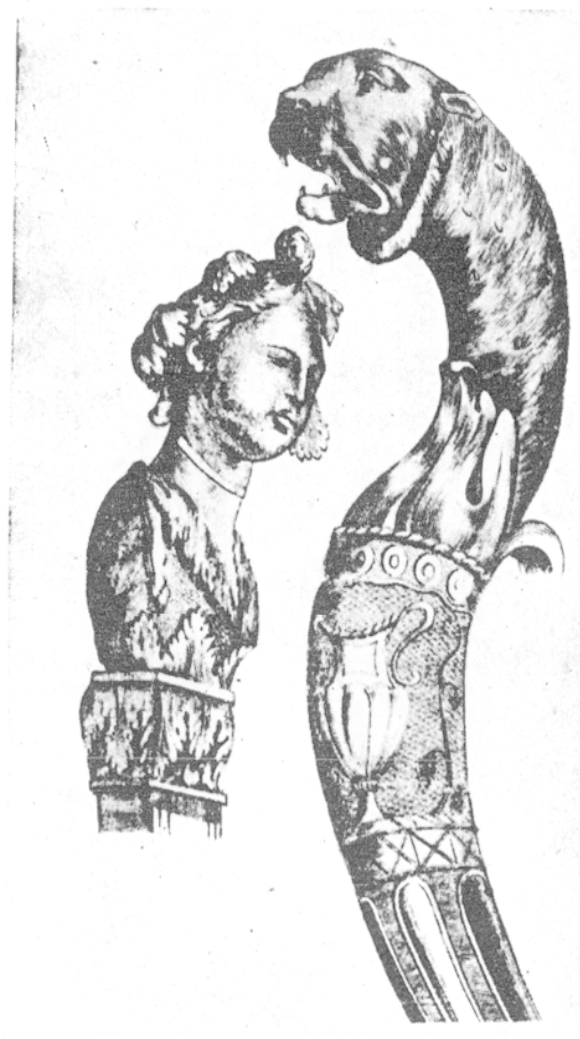

Fıg. 8. Détails du trépied de Bacchus.

D'après J.-B. Lambiez, ouv. cit., p. 231 sq. Cl. Appendice, noe 4, 5, 6, p. 189.

toutes sortes, des statuettes, des bronzes à tous usages ; une partie d'hypocauste avait été reconstituée dans le jardin du presbytère, où, par sucroit, en 1808, un bel aqueduc fut découvert (1). Comme tant d'autres avant et après elles, les collections d'Augustin Carlier furent perdues pour Bavai (fig. 9).

En 1813, J. de Bast publiait à Gand le second supplément à son Recueil $d$ 'Antiquités romaines. Il s'y était proposé, entre autres fins, d'étudier l'histoire de Bavai d'après les ruines qui se voyaient dans cette ville et les découvertes qu'on y avait faites (2). De celles-ci il dresse un consciencieux inventaire. C'est à lui que nous devons la première liste de marques de potiers trouvées à Bavai (3).

En 1825, une société se fondait à Valenciennes pour exploiter les champs de fouilles de Famars et de Bavai. Elle vécut deux ans. Deux ans de trop, dirait-on sans hésiter si l'architecte chargé de conduire les travaux n'avaif

(1) C.. le plan, en B.

(2) Cf. la préface, pp. 3 ss.

(3) Ouv. cit., pp. 53 ss. 


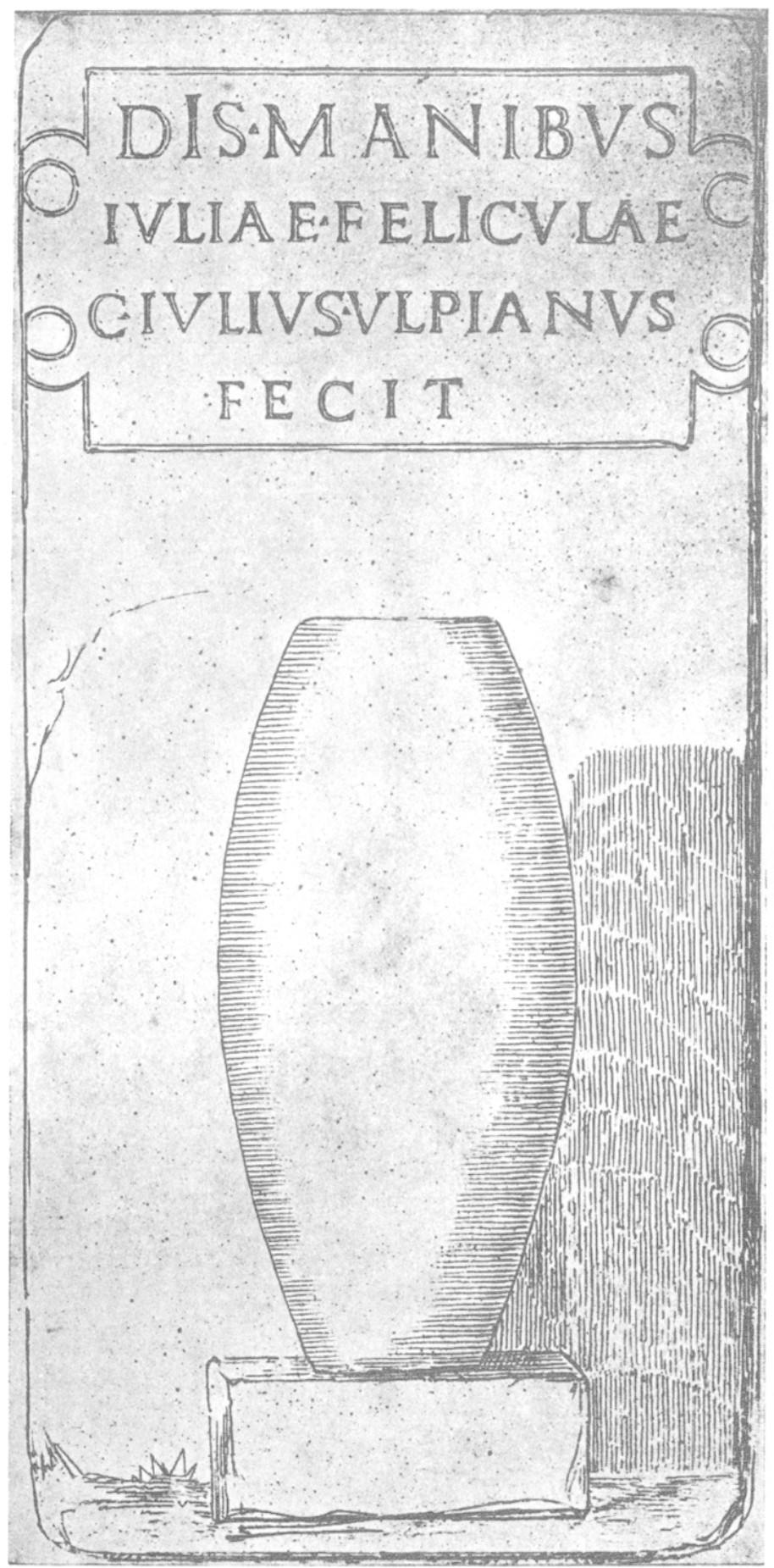

Fig. 9. Monument de Julia Felicula (hauteur, $1 \mathrm{~m} .28$; largeur, $0 \mathrm{~m} .63$; épaisseur, $0 \mathrm{~m}$. 45), découvert en 1777, provenant de la collection Carlier (aujourd'hui au Musée de Douai). Cf. Appendice, no 7, p. 189. 


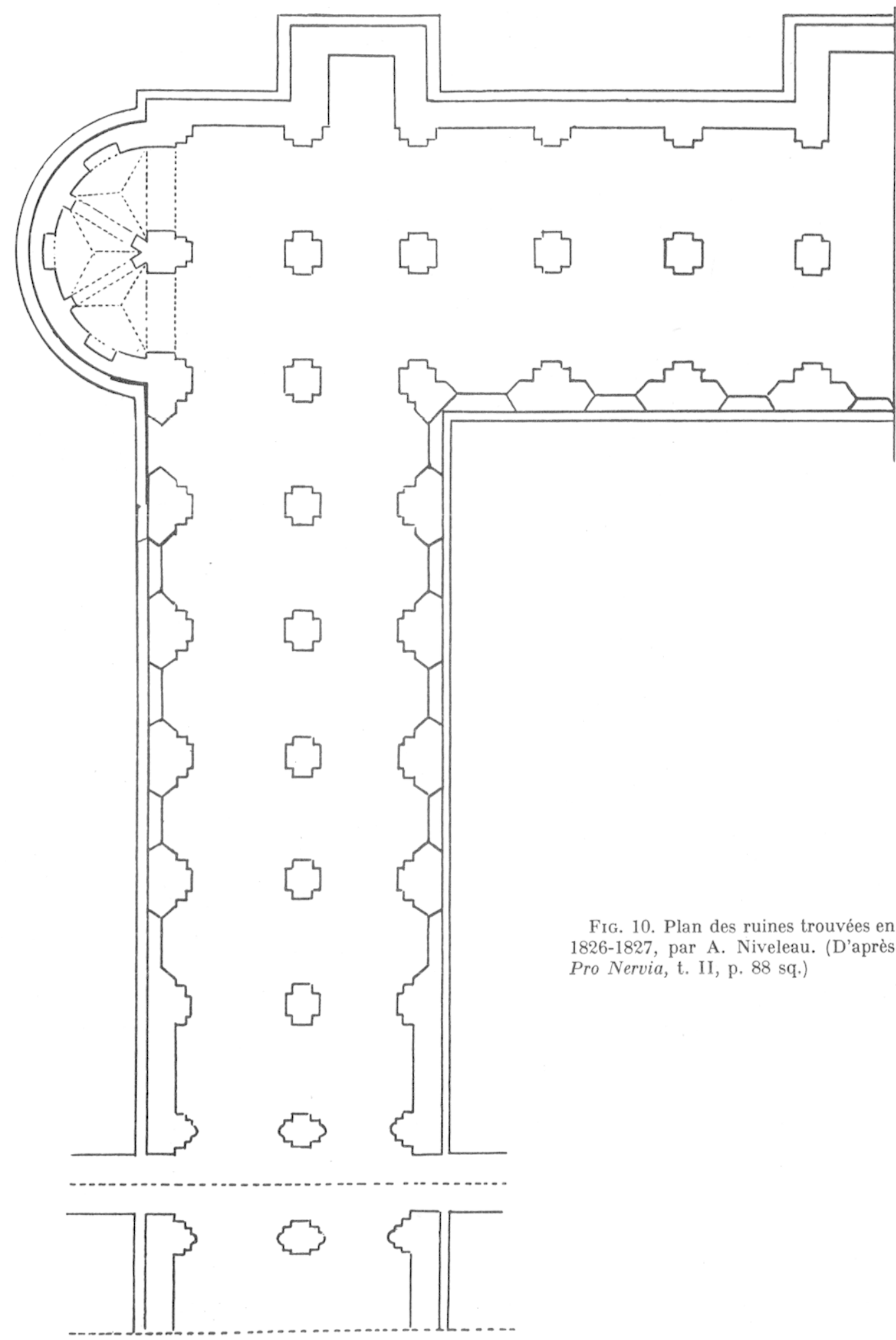


été Antoine Niveleau. Alors que les sociétaires ne pensaient qu'à se partager les objets découverts, lui, pendant les neuf mois que durèrent les fouilles, du 20 novembre 1826 au 30 juin 1827, relevait avec soin notes, croquis et plans (1).

Parti du pignon 0 . N. O. de la maison Bourlard-Durand, sise sur le Warechaix (2), il se glissa sous la courtine N. N. E. de l'enceinte gallo-romaine

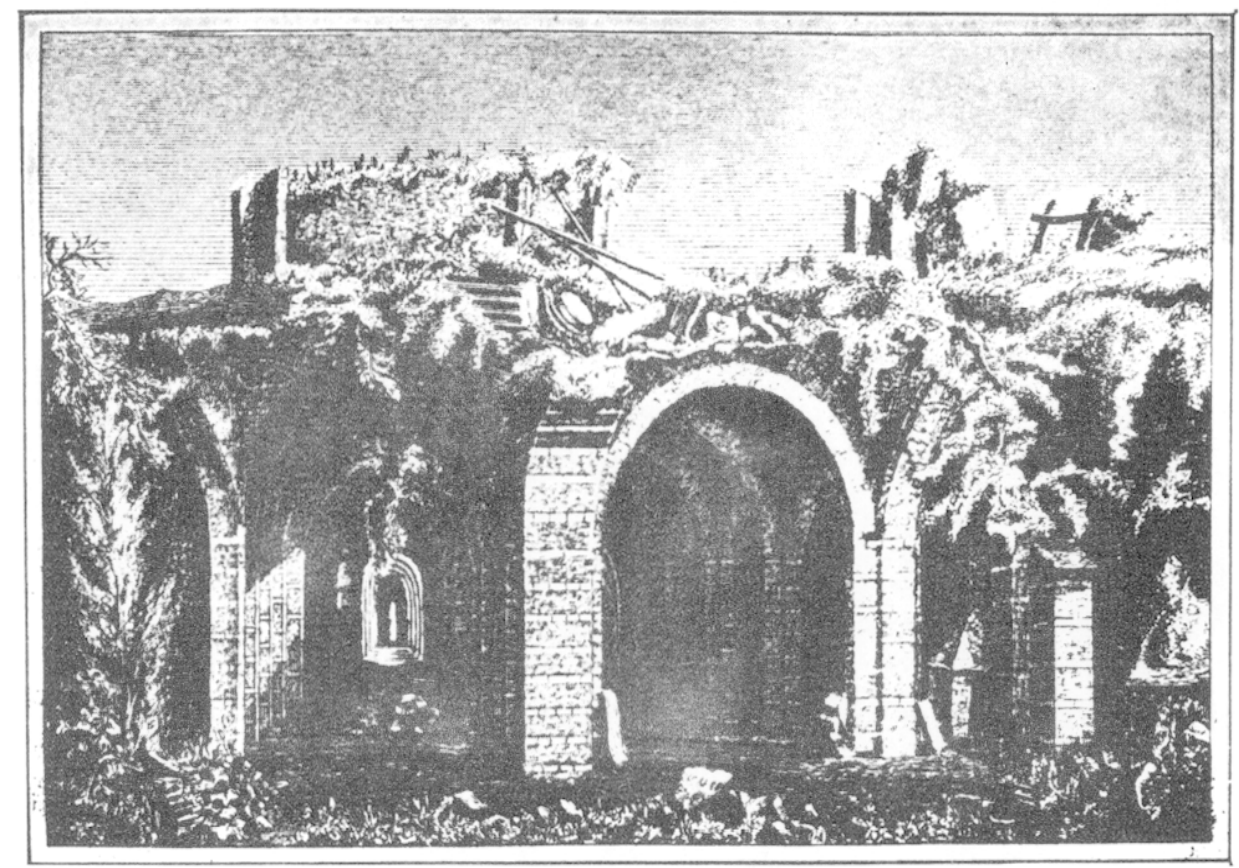

Fig. 11. Le c cirque de Bavai ", d'après une lithographie d'A. Prignet, illustrant l'étude de Lebeau sur Bavai (1844), Archives littéraires et historiques..., nouvelle série, t. VI.

jusqu'à une centaine de mètres plus à l'O. N. O. Au S. S. O. du souterrain il remarqua un mur à beau parement. Par la cave de la maison Bourlard-Durand il accéda d'autre part à un sous-sol gallo-romain. A quel édifice appartenaient donc le sous-sol et le mur à parement ? Les fouilles allaient le lui révéler.

Elles amenèrent en effet la découverte au S. S. O. de la courtine N. N. E. d'un ensemble de constructions anciennes, plus ou moins ruinées. Elles forment équerre (3) ; la partie parallèle à la courtine a 34 mètres de longueur ; l'autre, 45 ; elles sont larges de $13 \mathrm{~m}$. 50. Ce monument semble avoir comporté sous-sol et rez-de-chaussée, recoupés par une suite de piliers et voûtés (fig. 10).

(1) Ces documents forment les volumes manuscrits n. 604 et 605 de la bibliothèque de Lille.

(2) N. 286 du plan cadastral; cf. le plan, en C.

(3) Cl. le plan, en $D, D^{\prime}, D^{\prime}$. 
En dépit de ces découvertes, les doctes comme les profanes continueront longtemps encore à parler du " cirque » de Bavai. Tel Lebeau, en 1844, dans les Archives historiques el littéraires du Nord de la France et du Midi de la Belgique (1) (fig. 11). En 1847, il adoptera une autre interprétation, aussi contestable d'ailleurs : "En conférant le travail de Niveleau avec le traité de Vitruve, écrira-t-il alors, il m'a paru hors de doute que le monument élevé à Bavai l'avait été d'après les vues de cet architecte romain et que les ruines étaient celles d'un portique et d'un théâtre contigus (2)."

Le premier travail vraiment critique qui parut sur Bavai fut publié en 1873 dans les Mémoires de la Société d'Agriculture, de Sciences et d'Arts séant à Douai (3). Il avait pour objet, avec "Les voies romaines partant de Bavai au Ive siècle", les trois séries d'inscriptions - " monuments de pierres ", cachets d'oculistes, marques de potiers, - qui avaient été trouvées dans la capitale nervienne ou s'y rapportaient. L'auteur, Ernest Desjardins, intitulait l'étude des « monuments de pierre ": Monuments épigraphiques provenant de Bavai (4). Cependant l'un d'eux, conservé à Lyon, n'a pas été trouvé à Bavai. Il fut élevé par les trois provinces de Gaule au Nervien $L$. Osidius, qui, après avoir exercé toutes les charges dans sa cité, avait été prêtre de Rome et d'Auguste à l'autel du confluent (5).

De temps à autre, cependant, des découvertes se produisaient. En 1830, par exemple, on trouve sous trois maisons contiguës (6), rue Saint-Maur, les restes d'un hypocauste avec cent onze piles. En 1847, c'est le vase dit planétaire ou de Bavai, aujourd'hui au Cabinet des Médailles, que le sieur Bauchart exhume de son jardin, près de la chapelle Bronsin (7). La même année, à l'angle formé par la chaussée de Reims et la route d'Audignies, dans un coin où les trouvailles avaient déjà été nombreuses et notables, on recueille, avec divers débris architectoniques et sculpturaux, un groupe mutilé, en grès jaunâtre, qui représente un lion maintenant sous la patte gauche la tête d'un bélier, et, en grès également, une tête de jeune femme (8). En 1863, les restes d'un caveau funéraire sont trouvés chez les sœurs de Sainte-Thérèse (9), rue des Soupirs, et une colonne

(1) Nouvelle série, t. V, pp. 146 ss

(2) Archives historiques et littéraires..., nouvelle série, t. VI, p. 210

(3) Deuxième série, t. XI, pp. 81 ss.

(4) Ouv. cit., pp. 91 ss.

(5) C. I. L., XIII, 1702.

(6) N. $983,984,985$ du plan cadastral.

(7) P. Darche, Bulletin archéologique..., 1932-1933; "Les vases de Bavai ", p. 666, pl. XXII.

(8) Groupe et tête sont reproduits, sous les n. 3980 et 3982 , dans le Recueil général des bas-reliefs, statues et bustes de la Gaule romaine, par Espéravdiev, t. V, p. 189. Cf. les n. 3979, 3981, 3983 : autres pièces trouvées à Bavai.

(9) Plus précisément au-dessus des restes de ce caveau funéraire se touchent les parcelles n. 600 (immeuble des sœurs de Sainte-Thérèse), 607, 610. 
milliaire au bord de la chaussée de Reims, "à un kilomètre du centre de Bavai ". nous dit l'auteur de cette invention, Lucien Delhaye, dans son livre Bavai et la Contrée qui l'environne (1). En 1874, la construction de la gare amène la découverte de nombreux objets, dont deux situles en bronze, et la pose d'une voie de raccordement, celle de sept ou huit cercueils de plomb.

$\mathrm{Au}$ cours de la pramière moitié du XIx ${ }^{\mathrm{e}}$ siècle, avait commencé au S. $\mathrm{O}$. de la ville l'exploitation de sablières. Elle devait faciliter encore le travail des amateurs d'antiquités. "Le nombre de tous les objets trouvés à Bavai est vraiment prodigieux, écrivait L. Delhaye... Toutes ces antiques, qu'on compte par milliers dans chaque genre, se trouvent en ce moment déposées dans les cabinets particuliers de MM. Crapez, maire de Bavai, de Fourmestraul, maire de Gussignies, disséminées dans vingt autres habitations... (2) "Quand elle fut vendue, en 1881, la Collection Crapez comptait notamment huit monnaies gauloises, dont cinq en or, neuf monnaies romaines en or, quatre cachets d'oculiste et trente-huit statuettes en bronze (3).

\section{III. - De 1906 à 1942 .}

"Depuis des siècles, Bavai, la grande cité des Nerviens, est mise au pillage.» Ces mots de Franz Cumont, quand ils furent écrits, en 1914 (4), n'étaient plus tout à fait justes.

En 1906, M. Maurice Hénault, archiviste-bibliothécaire de la ville de Valenciennes, était venu pour la première fois à Bavai. Il avait étudié l'archéologie régionale et procédé à des fouilles préhistoriques et gallo-romaines dans le centre de Famars. Pendant trente ans il allait travailler à Bavai.

Il s'était d'abord préoccupé d'empêcher dans toute la mesure du possible la dispersion des antiques trouvées à Bavai. Avec le concours de quelques Bavaisiens, en 1907, il avait fondé un musée. Une chambre louée, rue Jordanez, abritait des collections particulières qui pour lors n'étaient que prêtées. Cependant M. M. Hénault et ses amis sont entrés en relation avec les propriétaires des sablières et les ouvriers qu'ils emploient. La couche de terre arable, plus ou moins épaisse, qu'il faut enlever pour atteindre le sable recèle en effet des restes d'habitation, des sépultures, des caveaux funéraires (fig. 12 et 13), des puits, des fours de potiers, où la récolte d'objets antiques est abondante. En 1909 les collections quittent la rue Jordanez pour être installées plus au large,

(1) Douai, 1869, p. 38 , n. 3 .

(2) Ouv. cit., p. 44, n. 1 .

(3) Catalogue de la Collection d'Antiquités romaines, gallo-romaines..., délaissée par M. A. Grapez.

(4) Ouv. cit., p. 79. 
place Jehan-Lemaire-de-Belges, dans le presbytère désaffecté, l'ancienne maison d'Augustin Carlier.

Certes il faut compter aven, certaines concurrences, certaines cupidités. Il arrive même que des pièces de grande valeur quittent encore Bavai. La loi

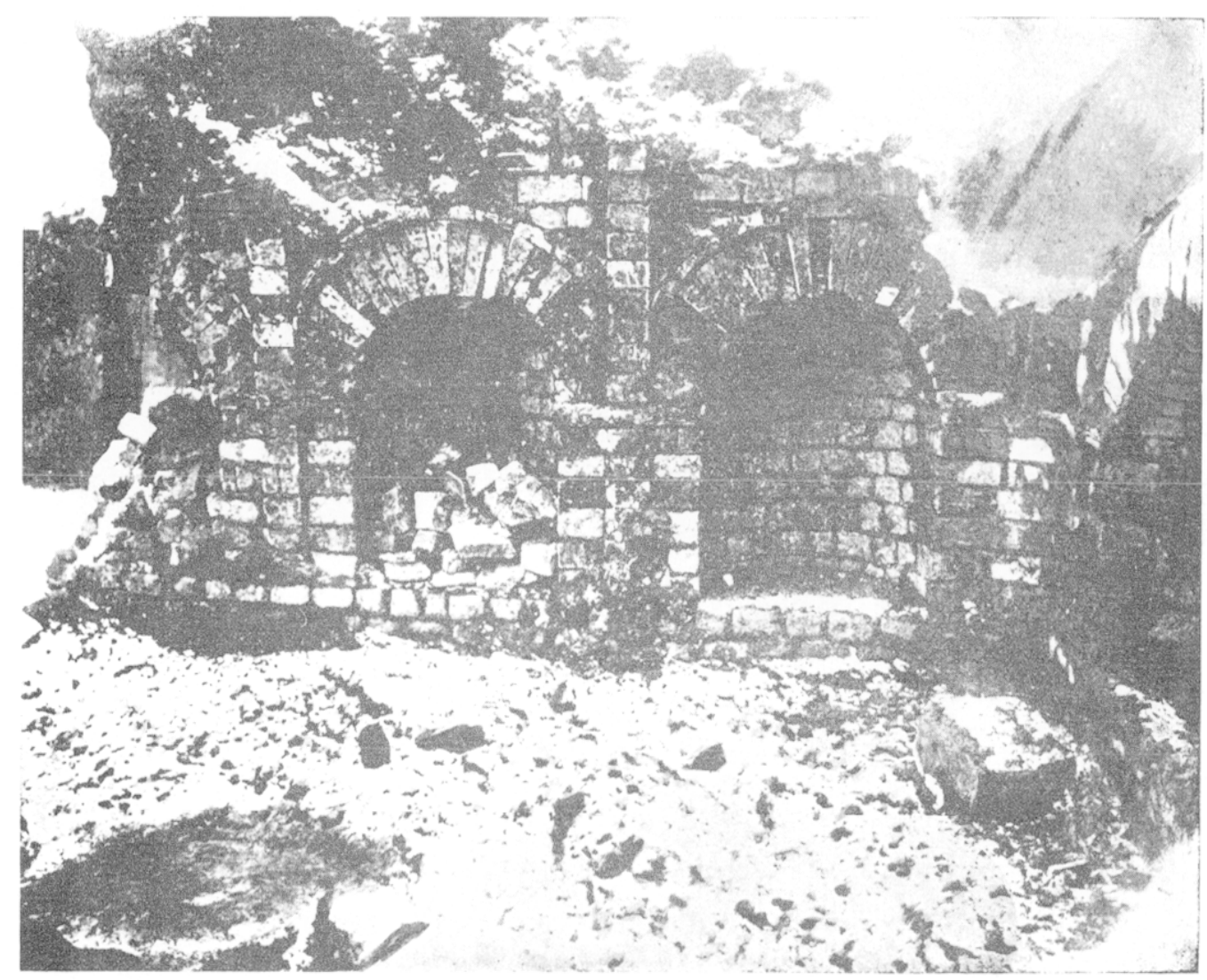

Fig. 12. Restes d'un caveau funéraire trouvés dans la sablière Lenglet. (Cliché M. Hénault.)

portant réglementation des recherches archéologiques et réservant au Pays le droit d'acquérir toute pièce intéressante se fera attendre jusqu'en septembre 1941. Cependant alors qu'en 1913 Espérandieu devait écrire : "La ville de Bavai, dont le musée est d'ailleurs de fondation toute récente, n'a rien conscrvé, ou à peu près, des antiquités sorties de ses ruines "(1), en septembre 1939, quand elles furent évacuées, les collections de ce musée comptaient 6.544 pièces.

Dans un local incommode, la présentation était telle qu'elle satisfaisait les connaisseurs et soutenait la curiosité du grand public.

(1) Ouv. cit., t. V, p. 187. 
FIg. 13. Ca-

ve d'habita-

tion trouvée

dans la sabliè-

re Denimal-

Mathieu. (Cli-

ché $M$. Hé-

nault.)
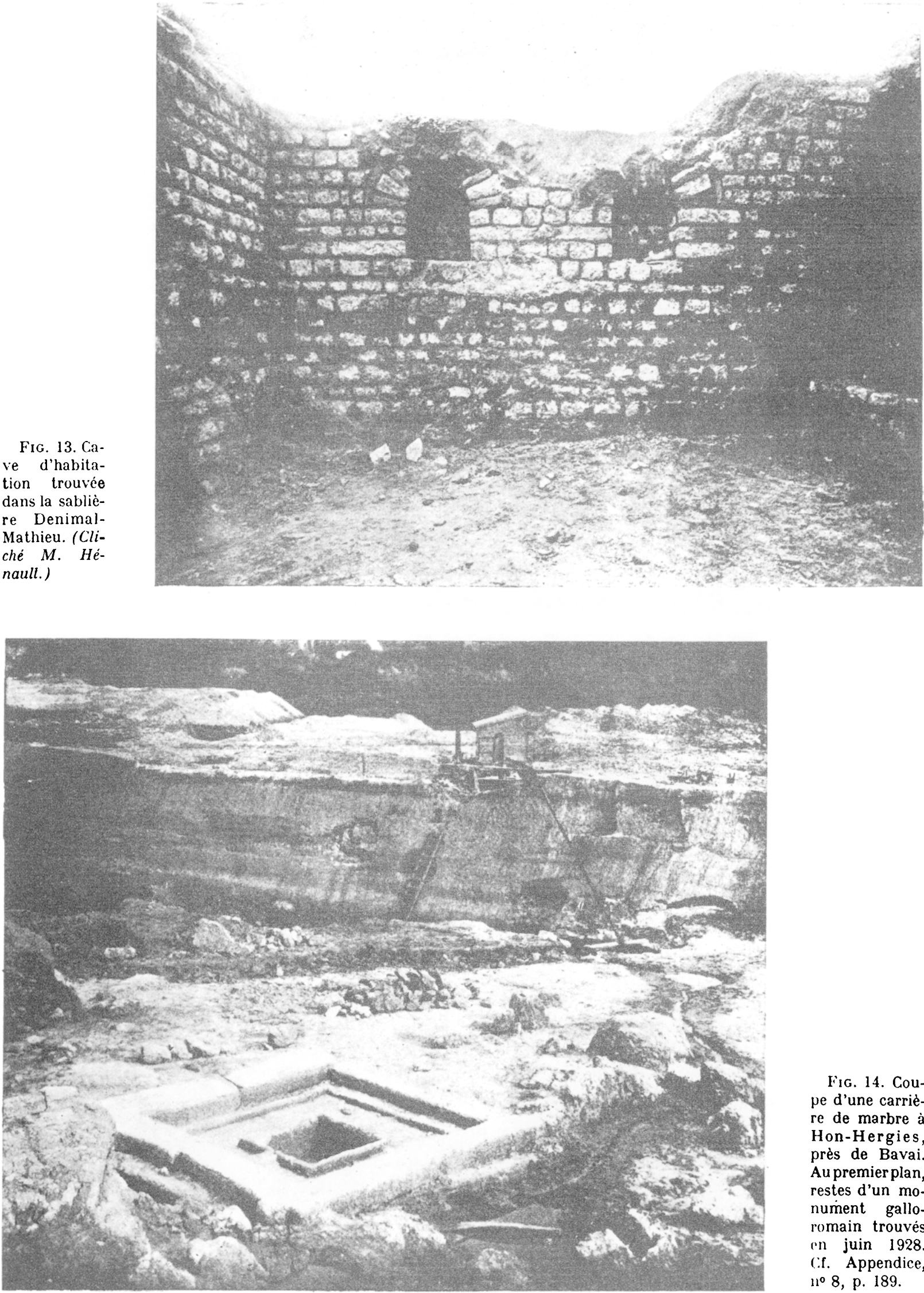

Fig. 14. Coupe d'une carrière de marbre à Hon-Hergies, près de Bavai. Au premier plan, restes d'un monument galloromain trouvés ('n juin 1928. C. Appendice, $11^{\circ} 8$, p. 189. 
Acquérir une trouvaille ne suffit pas ; il importe de noter avec soin toutes les conditions de la découverte. Avec autant de diligence qu'il le peut, M. M. Hénault

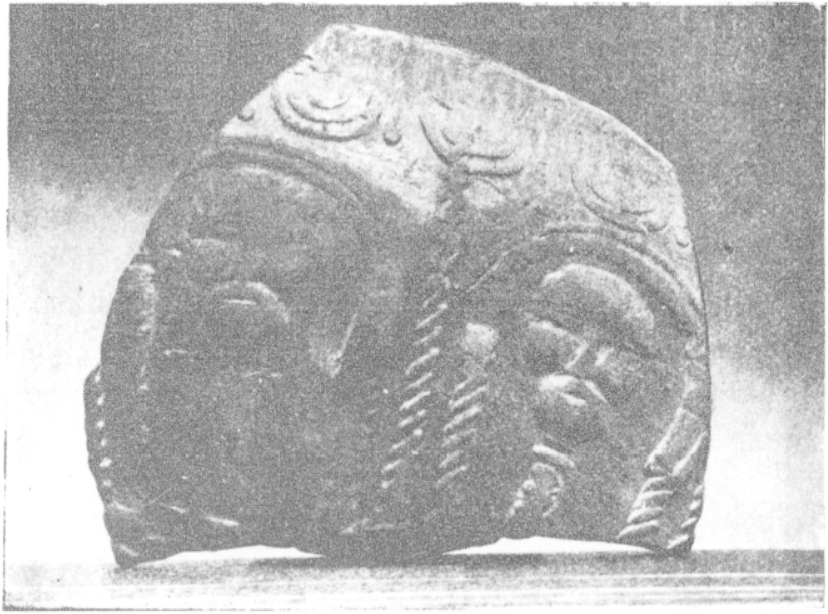

Fici. 15. Fragment de vase gallo-romain trouvé à Bavai, le 12 septembre 1923, dans la sablière Lenglet. (Cf. M. HÉvalti, dans Pro Nervia, t. II, p. 246 sq.) suit le travail des ouvriers dans les différentes sablières. Chacune de celles-ci devient un chantier de fouilles relativement peu coûteuses, où sans doute les exigences de l'exploitation et les intérêts de l'archéologie ne coüncident pas toujours, mais qui ménagent cependant l'occasion de recueillir un ensemble d'observations fort intéressantes.

Pour n'en donner qu'une preuve, à droite et à gauche de la route départementale $n^{0} 23$, qui relie la

frontière belge à Cambrai, les sablières se trouvaient sous l'emplacement de nécropoles antiques et Paul Darche, l'un des meilleurs auxiliaires de M. M. Hénault, dès 1923, écrivait : " L'exploitation des sablières, se poursuivant du plus loin au plus près de la ville, nous présente de précieuses coupes de terrain qui nous donnent la possibilité de fouiller dans leur ordre chronologique, sauf, parfois, quelques superpositions, une suite ininterrompue de sépultures allant de l'époque de La Tène III jusqu'aux environs du ve siecle (1)".

Des travaux de terrassement sont-ils effectués sur un autre point du territoire de Bavai ou des environs, M. M. Hénault en profite de même pour procéder aux observations utiles (fig. 14, 15, 16 et 17).

Si l'achat des trouvailles n'absorbe pas entièrement les crédits trop modestes dont il dispose, il prend à sa charge, pour travailler plus librement, l'enlevement des terres dans un coin des sablières

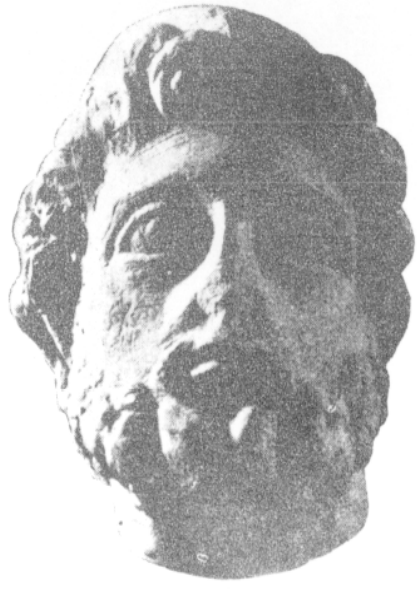

Fici. 1t. Tête difircule (?), trouvee dans la sabliere Lenglet. 


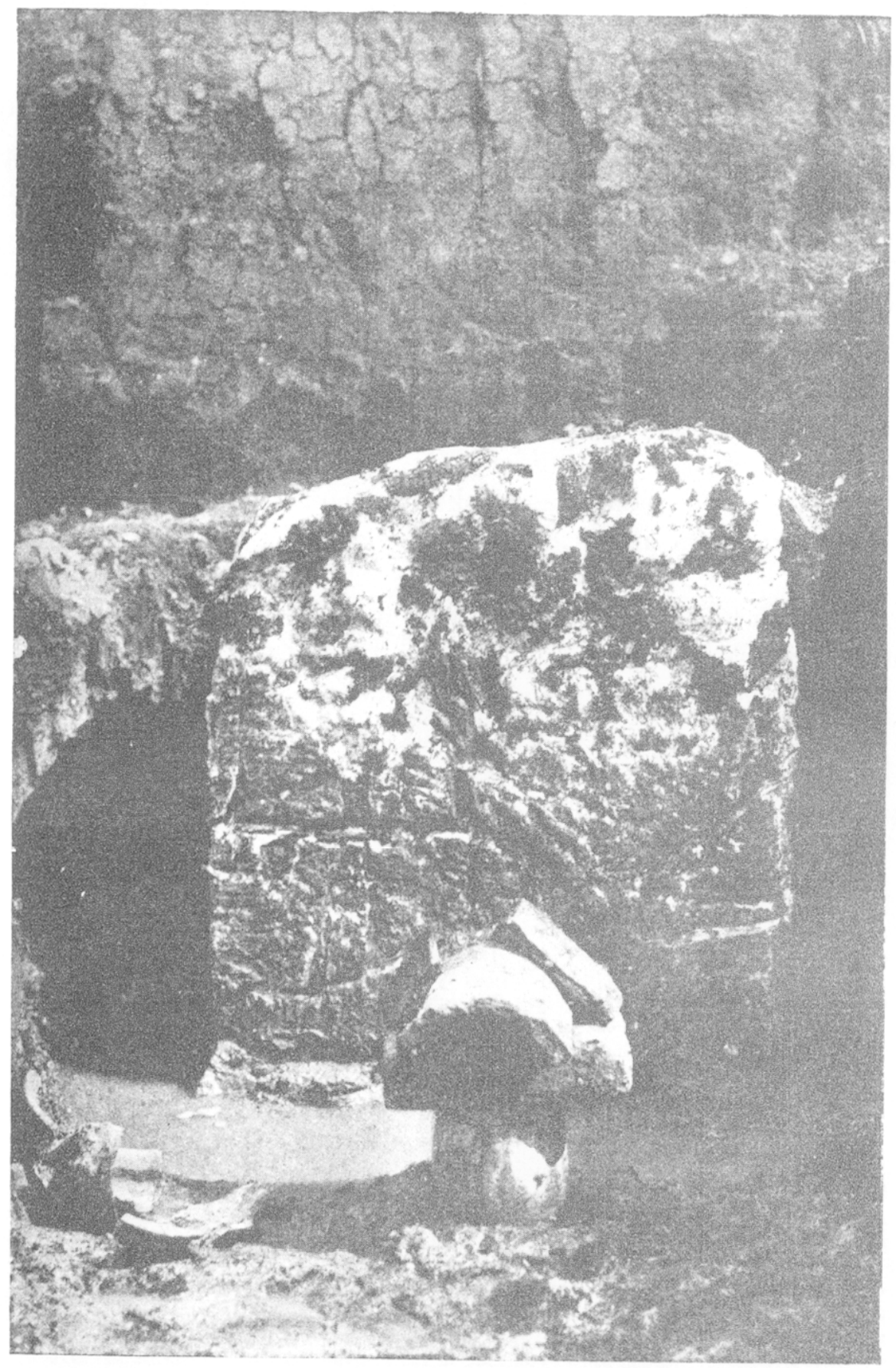

Fig. 17. Tombeau du ier siècle avec stèle et urne en plomb, trouvé en 1921, dans un banc de tourbe. (CP. Hénallt, Pro Nervia, III, p. 418 sq. Les sépultures à cercueils ou urnes de plomb à Bavai.) 
ou fouille quelque endroit intéressant. Hanté par le souvenir de Niveleau, M. M. Hénault recherche en particulier si le monument découvert en 1826 et 1827 se continue vers la courtine S. S. 0 ., puis parallèlement à elle.

Mais pour guider ces fouilles comme pour en apprécier les résultats, la connaissance exacte des découvertes réalisées dans le passé est indispensable. M. M. Hénault reprend donc pour le compléter et le poursuivre le travail de J. de Bast.

Il reste à publier les études dont ces recherches et les fouilles sont l'occasion et les conclusions qui s'en dégagent. M. M. Hénault lance en 1923 une revue, Pro Nervia. Il en est pendant onze ans le directeur et le principal rédacteur. En outre des rapports sur les fouilles et plusieurs études de M. M. Hénault et de P. Darche ont paru, de 1908 à 1937, dans le Bulletin Archéologique du Comité des Travaux historiques et scientifiques (1).

Il suffit de relever, dans ces publications, quelques faits pour se former une idée des résultats acquis grâce au travail de $M$. M. Hénault et de ses auxiliaires.

En octobre 1928, au delà de l'usine de palans, qui borde la chaussée d'Utrecht, un " atelier préhistorique " était découvert où l'on releva " de belles lames et des grattoirs certainement moustériens "(2). De 1933 à 1935, M. M. Hénault a " pu étudier en partie deux beaux ateliers, l'un dans la sablière Denimal, l'autre dans la sablière Dehon. Ces ateliers dataient tous deux de l'époque moustérienne, mais ont continué à être fréquentés, sinon occupés, à

(1) 1909, pp. 154 sq., M. M. Hénault : « Note sur une sépulture gallo-romaine découverte à LouvigniesBavai n, en novembre 1905 ; pl. XVII et XVIII ; cl. pp. 157 sq., HÉRON DE VILLEFosse : \& Les récipients de pierre en usage pour les sépultures à incinération »; pl. XIX. - 1911, pp. 221 sq., M. M. H... : OOiseau d'albâtre colorié trouvé dans les sablières de Bavai «, le 27 octobre 1910 ; pl. XVI et XVII. - 1914, pp. 49 sq., M. M. H... : " Nécropole antique à Bavai, rapport sur les fouilles de 1911-1912. " - 1922, pp. 15 sq., M. M. H... : * Caveau sépulcral du ${ }^{\text {er }}$ siècle à Bavai n, trouvé fin 1921 et début 1922 et pl. II à V. - 1928-1929, p. 493 ss., P. Darche : "Sur un fragment de vase orné du musée de Bavai " (scène de chasse, paysan portant un cucullus). - 1930-1931, pp. 521 sq. : "Les vases d'Albucius et de Cinnamus à Bagacum "; cf. p. 267. - 1932-1933, pp. 665 sq., P. D... : "Les vases de Bavai "; pl. XXII ; cf. p. 95 et 1934-1935, p. 384 ss. - 1934-1935, pp. 475 sq., M. M. H... : * Fouilles et découvertes à Bavai en 1934. * - Pp. 569 ss., P. D... : a Sur un rite funéraire et sur la rectification d'une lecture du Corpus ; cf. 19321933 , p. 341 et $1936-1937$, p. 83.

Cf. 1908, p. clviII, S. Reinach, sur la fondation du musée-bibliothèque de Bavai. - 1911, pp. xLvi sq., M. M. H..., sur le développement du musée. - 1912, p. XLiv, Héron de Villefosse, sur le musée. 1913, pp. Lvi sq., le même sur le rapport de M. M. H..., pour 1912 ; p. cxxxviI, le même sur les fouilles. 1919, pp. XLII sq., M. A. Blanchet, sur le musée pendant la grande guerre. - 1920, p. cLvi, M. M. H... sur un établissement céramique à Bavai. - 1924, p. xxv, C. Jullian, sur Pro Nervia, t. I, 1 et 2. - 1925, pp. Lv sq., M. A. BLANChET, sur le rapport de M. M. H..., pour $1920,1921,1922,1923$; p. cxxiv, P. D..., sur les marques de potiers du musée. - 1926, pp. cxxi ss., C. Jullian, sur Pro Nervia, t. I, 1-4 et t. II, 1-3. - 1927, pp. 173 sq., M. A. Blanchet, sur un journal de fouilles de 1925. - 1928-1929, pp. 104 sq., P. D..., sur des marques de potiers, pp. 116 sq., M. M. H..., sur la carte archéologique des arrondissements d'Avesnes, Cambrai, Valenciennes, pp. 117 sq., M. A. Blancher, sur une vue ancienne d'une partie de l'enceinte de Bavai. - 1934-1935, pp. 292 sq., P. D..., sur un coin intéressant des nécropoles de Bavai. - 1936-1937, pp. 82 sq., P. D... : "Les dieux de la semaine sur les vases de Bavai », pp. 259 sq., M. M. H..., sur la voie Bavai-Reims.

(2) P. N., t. V, p. 53. 
des époques postérieures, surtout à l'époque tardenoisienne. Nombreuses sont aussi les haches polies, entières ou en fragment, qui ont été recueillies sur le territoire de Bavai comme sur ceux qui l'environnent. Sur l'emplacement de la sablière Dehon, des grès énormes ont été rencontrés; certains d'entre eux dressés et non couchés faisaient penser à des menhirs " (1).

En juillet 1921, M. L. Desailly avait publié dans le Bulletin de la Société préhistorique française une étude intitulée : "Les chaussées romaines de Bavai n'ont-elles pas une origine préhistorique? " où il remarquait que les rares monuments mégalithiques connus dans la région de Bavai se trouvent pour la plupart érigés près des chaussées qui aboutissent à cette ville (2).

Pour l'âge du Bronze, M. M. Hénault fait état dans Pro Nervia (3), des quarante objets acquis en 1852 à Bavai par le comite de Robiano et que mentionne Déchelette dans son Manuel d'Archéologie Celtique ou protohistorique (4).

De l'époque gauloise les fonds de cabanes et les fours de potiers que M. M. Hénault a fouillés et les multiples objets qu'il a recueillis prouvent que le plateau où s'élève

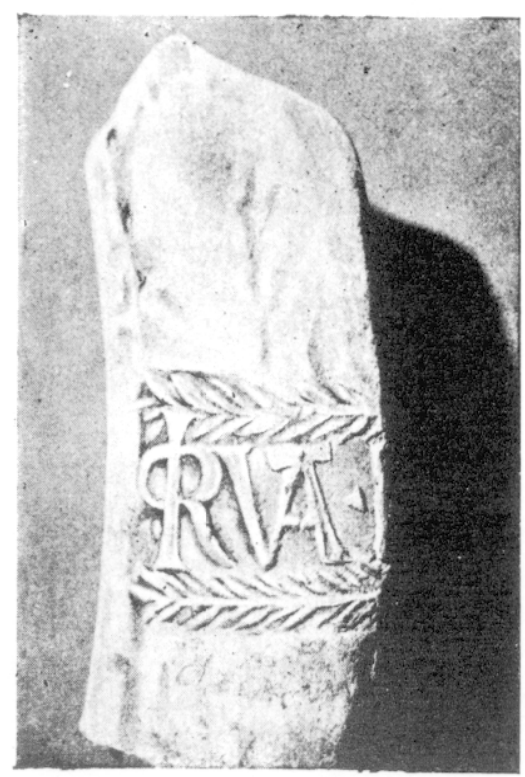

Fig. 18. Fragment de bord de pelve avec marque du potier PRIVAT [V S], cf. Appendice, no 9, p. 189. (Fouilles Biévelet.) Bavai n'a pas cessé d'être habité entre l'époque protohistorique et la conquête romaine.

A l'aide des trouvailles particulièrement abondantes de vases et de fragments de poteries, M. M. Hénault et $P$. Darche peuvent étudier l'industrie et le commerce de la céramique, à Bavai, sous la domination romaine. Ils montrent, par exemple, que la lignée des Brariati s'espace entre 86 avant notre ère et la veille de la grande invasion de 276 ; qu'ils écoulaient leurs produits principalement le long des grandes voies qui se dirigent vers le Nord, celles de Bavai à Cologne et de Bavai à Utrecht; que l'un ou l'autre d'entre eux eut en dehors de Bavai un centre de fabrication (5) (fig. 18).

(1) P. N., t. VII, pp. 10 sq.

(2) C.. P. N., t. V, p. 105.

(3) P. N., t. VII, p. 11 .

(4) Appendice I, n. 582.

(5) P. N., t. I, pp. 114 sq. Cl. ibid., t. VI, p. 14, une opinion plus réservée de P. Darche sur les dates extrêmes de cette " firme " des Brariati. 


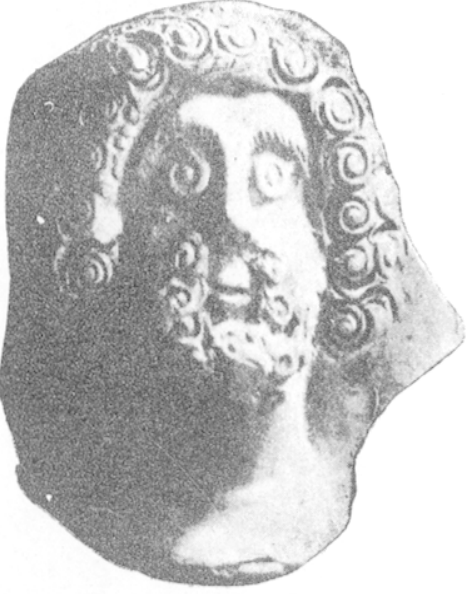

Fig. 19. Fragment de vase de Bavai au Musée de Lille.

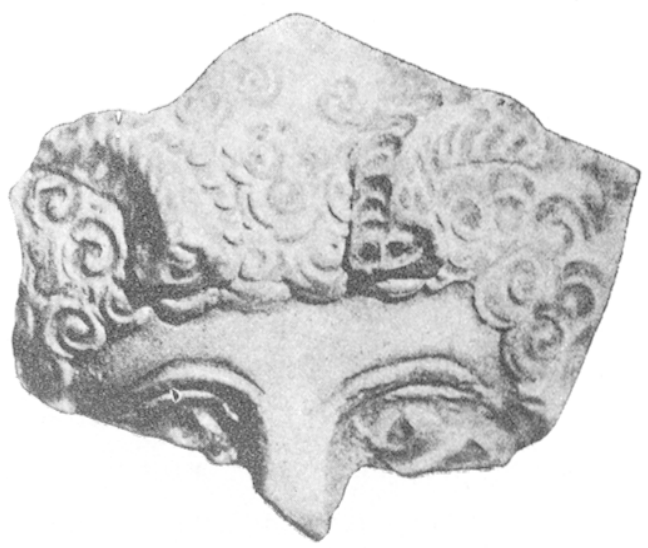

Fig. 20. Fragment de vase de Bavai trouvé le 11 avril 1940.

Ils établissent de même que les potiers de Lezoux Albucius et Cinnamus, de l'époque Adrien-Antonin le Pieux (117-161), " avaient à Bagacum des représentants si bien "introduits " dans la clientèle locale qu'ils en étaient arrivés à leur assurer la presque "exclusivité » de la vente des vases ornés » (1).

A propos des vases " dont les parois figurent en relief les sept dieux planétaires "Camille Jullian écrivait, en 1920, au tome V de son Histoire de la Gaule : "Ces vases n'ont guèrc été fabriqués qu'en Belgique chez les Tongres

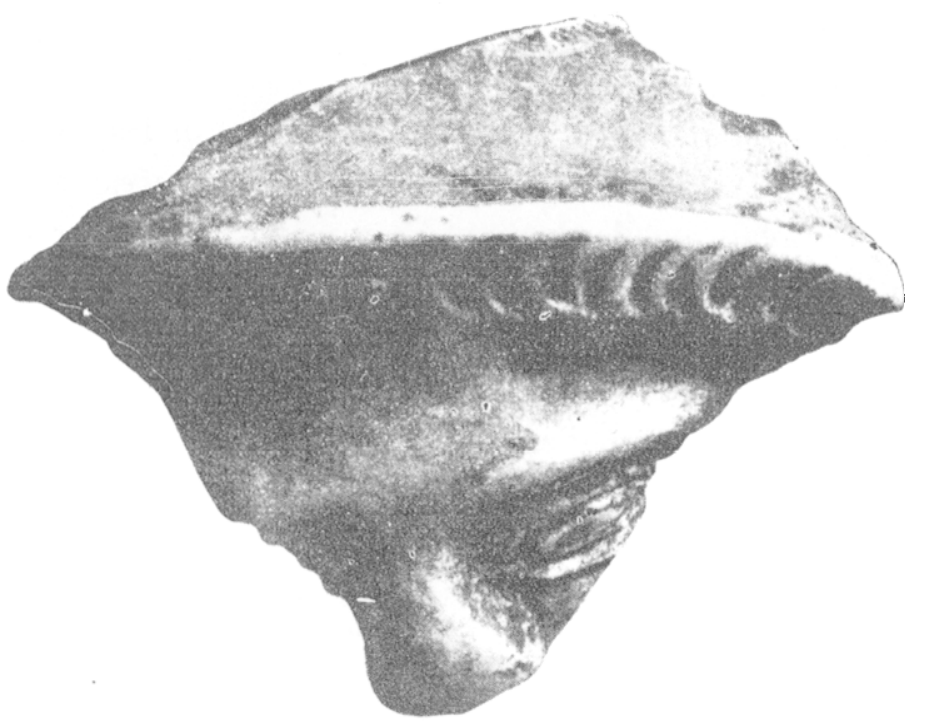

Fig. 21. Fragment de vase trouvé le 9 juillet 1942. ou les Nerviens, et à la fin du iI ${ }^{e}$ siècle au plus tôt; jusqu'ici on n'en connaît qu'un petit nombre (2). "Or le 9 décembre 1935, rendant compte à la Section d'archéologie du Comité des Travaux historiques et, scientifiques d'un mémoire sur les Vases de Bavai de Paul Darche, M. Lantier constatait avec ce-

(1) P. N., t. VI, p. 81 .

(2) p. 271, n. 8. 
lui-ci que la dispersion des trois vases planétaires et des cent neuf " tessons appartenant, à la même catégorie de représentations " est telle qu'elle " parle en faveur de l'hypothèse plaçant à Bavai le centre de fabrication de cette céramique historiée ". "Pour confirmer cette hypothèse séduisante, ajoutait-il, il ne reste qu'à souhaiter la découverte de l'officine dans laquelle on cuisait cette curieuse céramique... La confrontation des monnaies et des marques de potiers étrangers à la région recueillis avec les vases planétaires... permet (à M. P. Darche) de serrer d'assez près la chronologie de cette poterie qui appartient aux I ${ }^{\text {er }}$ et II ${ }^{\mathbf{e}}$ siècles de notre ère et disparaît à l'époque des Antonins (1) (fig. 19, 20 et 21).

Des " sondages ", opérés en 1910, 1921, 1922-23, démontrent que les constructions du monument retrouvé par Niveleau s'étendent parallèlement à la courtine S.S. O. sur plus de 110 mètres de longueur (?). M. M. Hénault incline à voir dans ces ruines les restes d'une basilique qui entourait de trois côtés une place, sans doute le forum (3). Au gré de Camille Jullian, particulièrement sévère pour l'œuvre de Rome dans les Flandres et les Ardennes, Bavai était une " bourgade " (4), une "petite ville " (5). A supposer qu'il eût raison, on imagine l'effet que devait y produire un édifice de pareilles dimensions.

Des fragments de poteries et deux sépultures ont permis à M. M. Hénault d' " affirmer " que cette "basilique " avait été en bonne partie détruite avant le rve siècle (6).

Entre 1911 et 1923, enfin, il a pu fouiller, au Brai Préchon dans la forêt Mormal, quatorze tombes de l'époque carolingienne disposées au milieu de substructions gallo-romaines (7).

Depuis 1936, les recherches, sous la direction du signataire de ces lignes (fig. 22), ont porté principalement sur la " basilique ". A l'E. S. E. de celles qu'avait reconnues M. M. Hénault en 1922-23

(1) Bulletin archéologique..., 1934-1935, pp. 384 sq.

(2) $P$. N., t. I, pp. 148 sq. Cf. le plan : en E, E', E', sondages de 1910 ; en F, sondages de 1921 ; vers le point $G$, sondages de 1922 1923. En outre, M. M. Hénault a procédé à des recherches près de la tour 5 , au nord, en 1911 ; près des tours 2 et 3 , en 1922 et $1923 ; \dot{\delta}$. l'est de la tour 5 , en 1928 ; vers le point $H$, en 1929 ; vers le point $I$, en 1932 et 1933 . Cf. $P$. N., t. V, pp. 37 sq. ; t. V, pp. 83 ss., et du même, " $A$ la recherche du Monument », inédit.

(3) P. N., t. VI, pp. 67 sq. ; t. VII, p. 19

(4) Histoire de la Gaule, t. VI, p. 460.

(5) Ibid., p. 467.

(6) P. N., t. I, pp. 161 ss.

(7) P. N., t. I, pp. 167 ss.

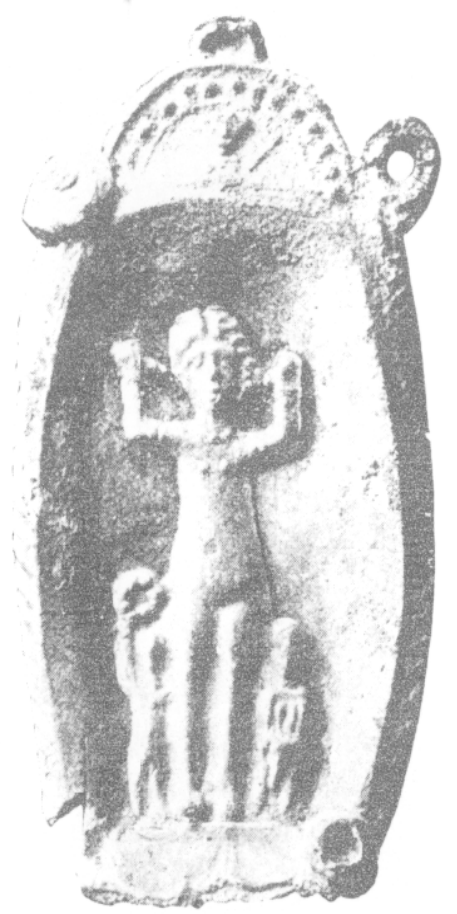

FIG. 22. Amulette de plomb acquise en 1936, trouvée à Louvignies (hauteur, $0 \mathrm{~m} .085$; largeur, 0 m. 042 ; poids, 72 grammes). 
d'autres substructions furent dégagées, en 1938-39, sur $27 \mathrm{~m} .2$ (1). A l'aplomb de la paroi intérieure du mur d'enceinte et juxtaposés sur un mur arasé se trouvaient trois fragments d'un bas-relief, restes d'une frise sculptée (fig. 23, 24 et 25). Plusieurs des deux cent vingt objeis qui furent recueillis au cours de ces fouilles avaient subi l'action du feu ; certains étaient réduits à l'état de masse informe de métal.

Dès lors la Direction des Monuments Historiques était résolue à mettre
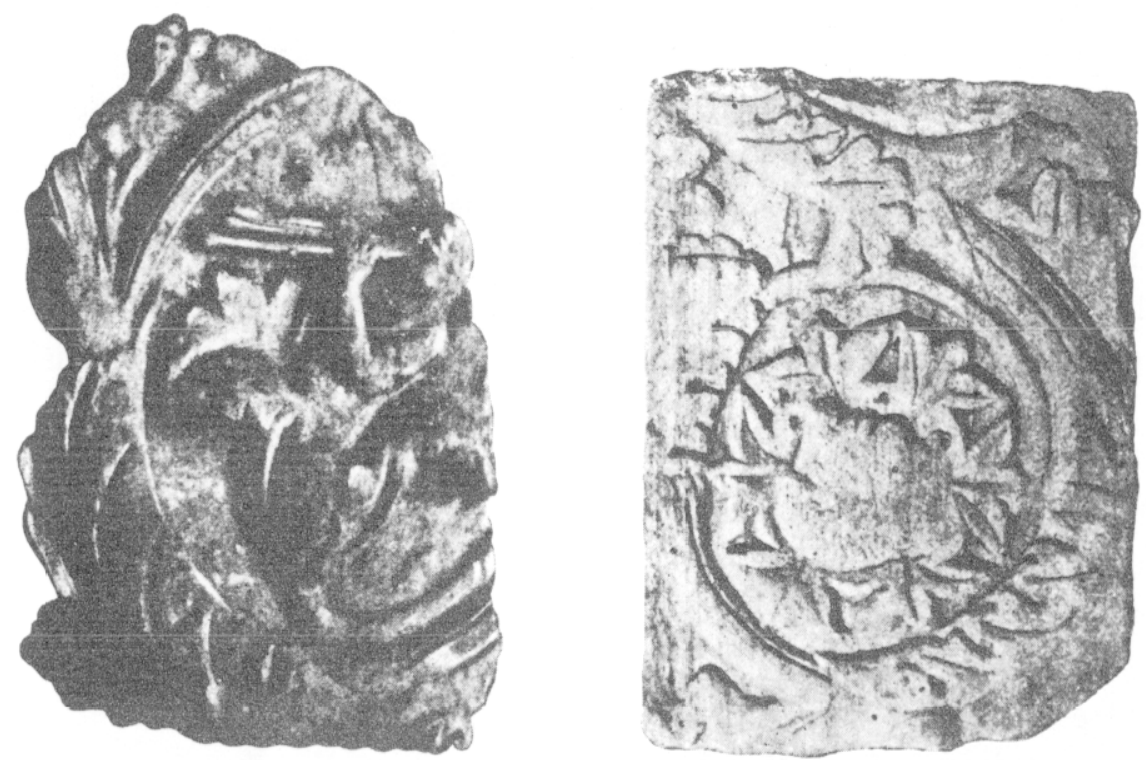

FIG. 23-24. Fragments d'une frise sculptée.

au jour, sur une longueur de 130 mètres environ à partir du front 0 . N. O. de l'enceinte, la "basilique " et le "forum ".

Le 17 mai 1940 les coups de la guerre, une fois de plus, s'abattaient sur Bavai. Si les ruines gallo-romaines étaient épargnées, des 467 maisons que compte la localité, 140, hélas !, étaient détruites et 87 autres, bien endommagées. Des fouilles devenaient possibles dans les terrains ainsi brutalement déblayés (fig. 26 ct 27).

En mars et avril 1942, à 180 mètres de la partie O. N. O. de la "basilique ", était trouvée une suite de piliers conservés sur une hauteur maximum de $1 \mathrm{~m} .90$. Elle est coupée par une rue et cependant douze piliers ont pu être mis au jour. Ils ont $1 \mathrm{~m}$. 50 de largeur et, s'élevant à $3 \mathrm{~m} .50$ l'un de l'autre, ils s'espacent

(1) Cl, le plan en J, 
sur une ligne de 70 mètres. Ils sont formés de lits de pierre blanche et de pierre bleue, en petit appareil, et de lits de tuileaux (fig. 28). Près de ces piliers. à l'E. S. E. comme à l'O. N. O., des murs furent aussi découverts (1). De même trois sondages opérés sur l'emplacement présumé de l'aile N. N. E. de la "basilique ", à l'E. S. E. de la maison Bourlard-Durand, révélaient des substructions et un pan de la muraille d'enceinte renversé (2).

Cependant près de la porte de Valenciennes, entre la route nationale no 49 ,

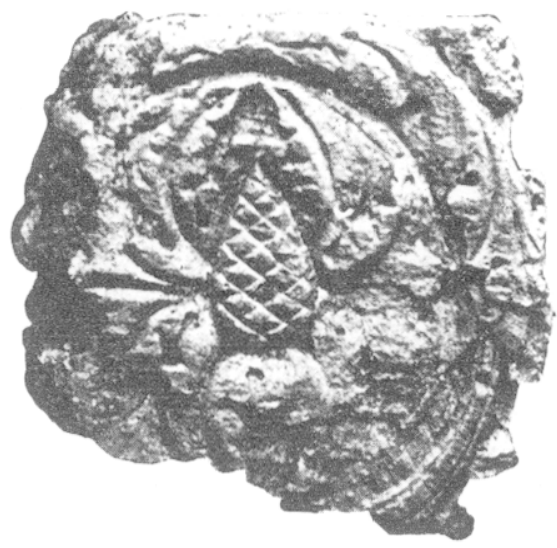

Fig. 25. Fragment d'une frise sculptée.

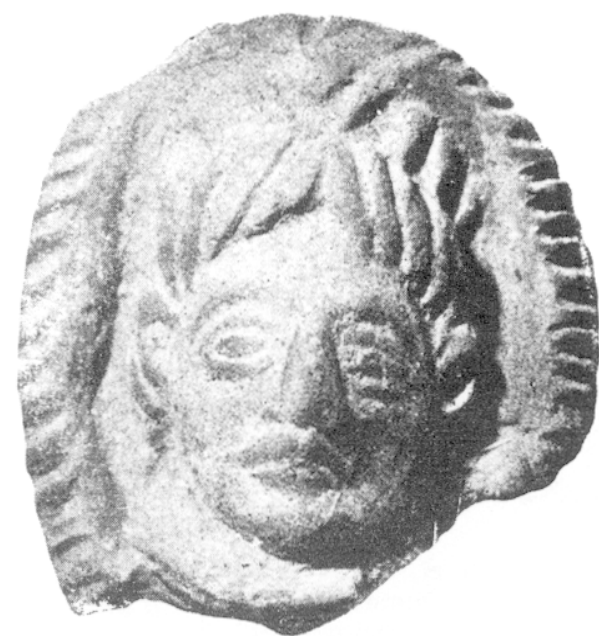

FIG. 26. Tête, poterie rouge à pigmentation dorée. (Fouilles de 1940.)

de Valenciennes à Maubeuge, et la route de grande communication $\mathrm{n}^{\circ}$ 24, de Gussignies à Avesnes, une cité de relogement était aménagée. Les terrassements amenèrent la découverte des restes d'un hypocauste et de substructions entourant une aire de 90 mètres carrés, encore à fouiller ( fig. 29).

Dans sa séance du 4 juillet dernier, la XVe Commission du Centre national de la Recherche scientifique, sur un compte rendu de M. Jean Verrier, inspecteur général des Monuments historiques, adoptait toutes les conclusions d'un rapport, daté du 9 juin, de M. René Louis, directeur de la première circonscription archéologique : ainsi se trouvaient décidés : 1) le dégagement des plliers découverts

(1) Cf. le plan : en $\mathrm{K} \mathrm{K}^{\prime}$, les piliers ; en L et $\mathrm{M}$, murs ; en $\mathrm{N}$, aqueduc (?).

(2) Ci, le plan : en $\mathrm{O}$ et $\mathrm{O}^{\prime}$, substructions ; en $\mathrm{O}^{\prime \prime}$, pan de muraille de l'enceinte renversé. 


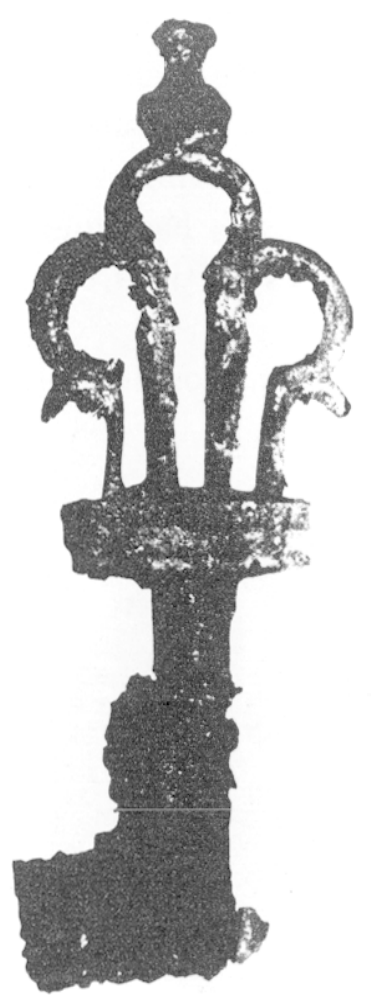
en 1941. (Le panneton est en fer; le reste en bronze.) Cr. Appendice, no 11, p. 189.
Fic. 27. Clef trouvée

en mars-avril ; 2) la délimitation d'une zone archéologique au N. N. O. de la ville et le classement des terrains de cette zone; 3 ) le classement de vestiges qui se trouvent en dehors de cette zone ; 4) la reprise en sous-œuvre d'une partie de l'enceinte gallo-romaine qui, faute de fondations solides, menace de s'écrouler; et 5) des fouilles sous l'église, incendiée le 17 mai 1940. A la demande de M. J. Verrier, lả Commission approuvait en outre l'établissement d'une zone de protection au sud de la zone archéologique (1).

Un nouveau musée a été prévu pour recevoir avec les collections déjà rassemblées les futures acquisitions.

Reprenant avec plus de méthode et de science le travail des siècles passés, les recherches des quarante dernières années avaient montré l'importance du centre archéologique de Bavai. Ces mesures intervenaient opportunément pour mettre celui-ci sans plus tarder en valeur.

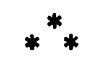

“Les Francs d'abord, puis les Vandales ont détruit Bavacum... Si bien qu'à Bavai même aujourd'hui il nous faut .chercher Bavacum. "Ainsi parlait Aubert Lemire cn 1624 (2).

Depuis, trois siècles ont passé, durant lesquels les traces de Bavacum ont continué à s'effacer et ses ruines à périr. Le jour cependant semble s'annoncer ou, en partie du moins, les vestiges de la capitale de la Nervie auront été retrouvés.

Henti BiÉvelet.

(1) Cf. le procès-verbal de la séance.

(2) Rerum Belgicarum Annales, p. 41 : "Bavacum porro Franci primum vastarunt, deinde Vandali... Adeo quidem ut in ipso Bavaco hodie Bavacum quæramus. "

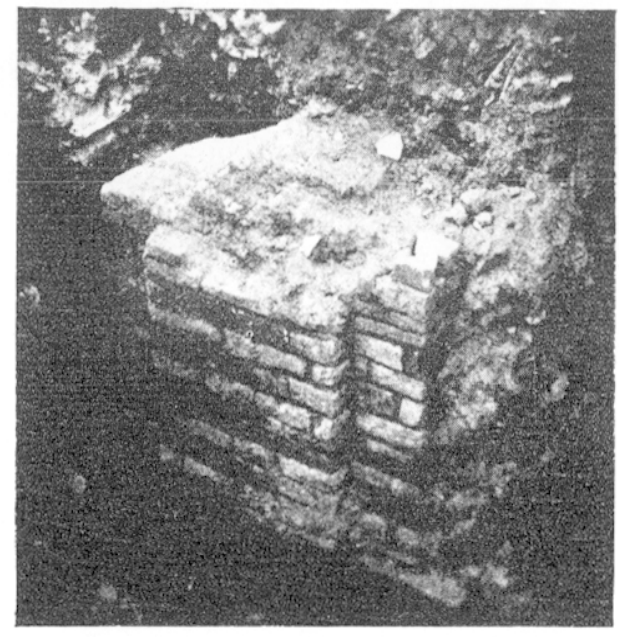

Fıg. 28. Base de pilier trouvée le 10 avril 1942. 


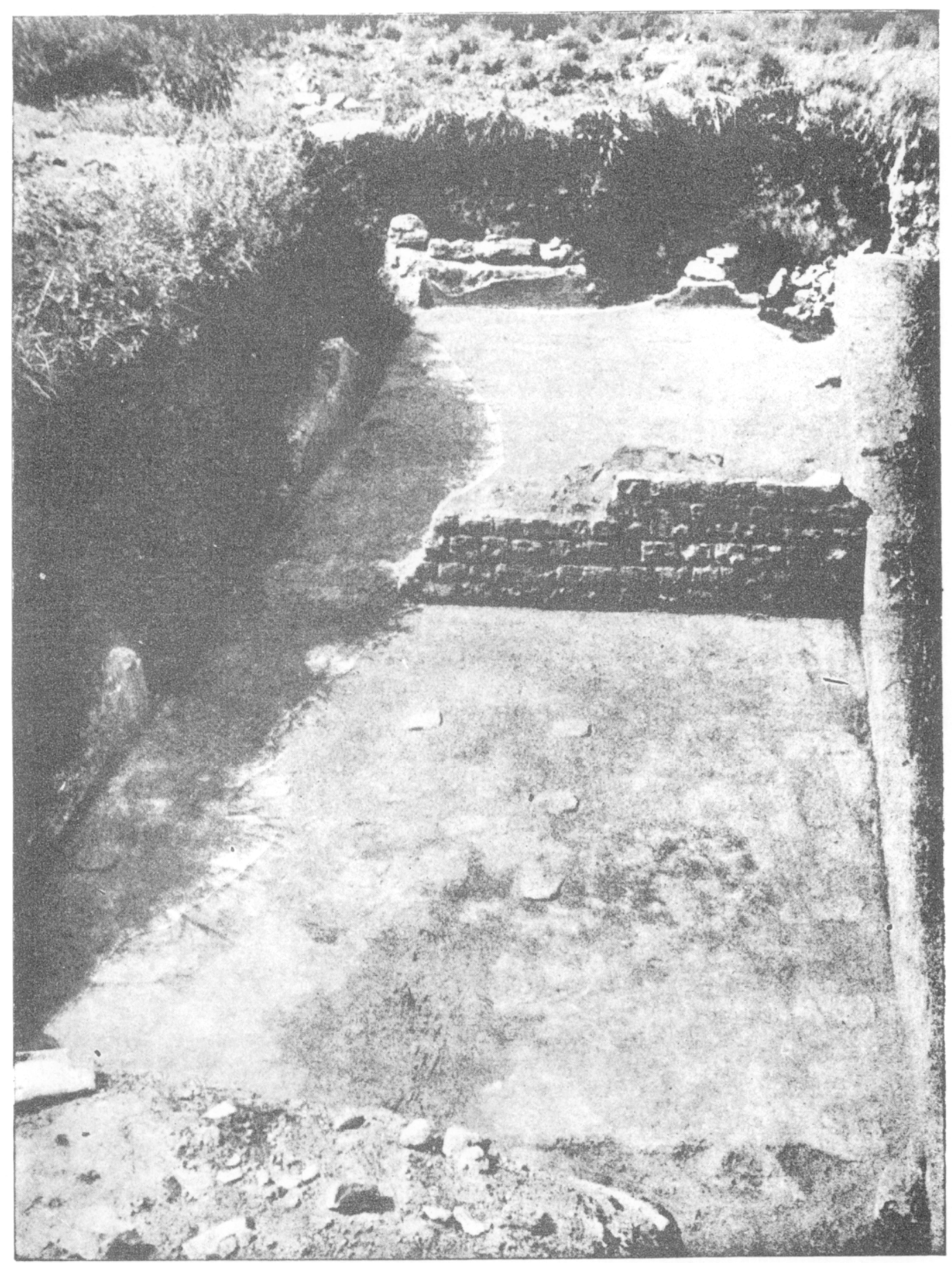

Fig. 29. Restes d'un hypocauste trouvés en 1942. Cf. Appendice, n 11, p. 189. 


\section{APPENDICE}

\section{Observalions sur quelques-unes des figures reproduites ci-dessus}

(1) De Caylus, ouv. cit., p. 398, écrit : "Ce morceau a été bien jetté (sic). Sa longueur est de six pouces et demi, et la plus grande hauteur, de deux pouces, neuf lignes. " Franz Cumont pourra dire : "Une série dé poignées de commode ou de coffret, décorées de bustes de Cybèle accostée de ses lions, et d'Attis avec la pomme de pin qui lui est consacrée, ont été mises au jour à Bavai et aux environs et l'aire des trouvailles comme le style de cet objet d'ameublement ne permettent pas de douter que le chef-lieu de la Nervie ait fourni ces poignées en quantité considérable aux châtelains d'alentour "(Comment la Belgique fut romanisée, dans Annales de la Société royale d'Archéologie de Bruxelles, t. XXVIII, 1914, p. 147. Cf. ibid., fig. 46).

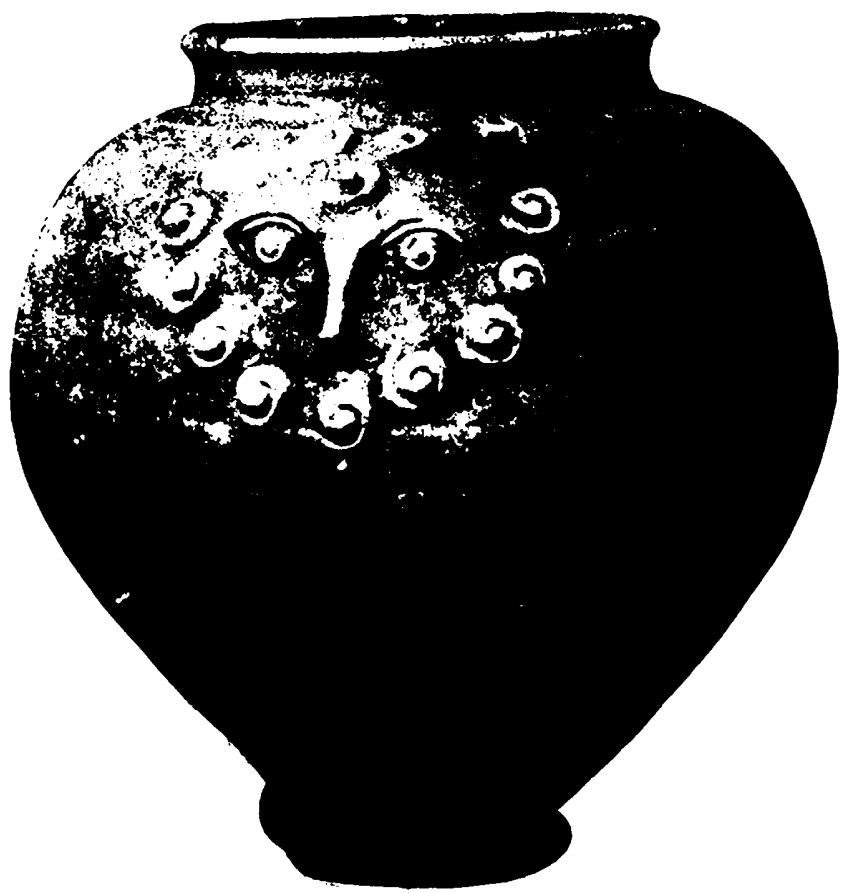

Vase en poterie grise (Musée de Bavai).

(2) De Caylus, ouv. cit., pp. 395 ss., écrit : “ Les caractères de l'inscription ne peuvent être plus beaux; ils sont du meilleur temps des Romains... Ce petit monument a encore onze pouces de hauteur, sept de largeur, et trois d'épaisseur. „Le C. I. L., XIII, 3569, propose de lire : "Nervinis [Matribus]. „Ce cippe est maintenant au Cabinet des Médailles.

(3) De Caylus, ouv. cit., p. 399, écrit : « Cet ouvrage de mosaïque, que M. de Lucé, alors Intendant de Valenciennes, a fait dessiner avec soin sur les lieux, mérite d'être rapporté. Voici ce qu'il écrivit au Ministre dans le temps de la découverte : * Au mois de mai 1751, un particulier de Bavai, en tirant des - pierres de quelques fondations anciennes, a trouvé dans son jardin le morceau de mosalque, dont je joins - ici le dessein. Il a près de treize pieds de longueur sur un peu plus de huit de large. Les cubes de cette - mosaique sont de pierre, et ils ont environ quatre lignes en quarré, et quelques-uns quatre lignes sur - sept. Le mastic, ou plutót le ciment, dans lequel elles sont posées, a plus de cinq pouces d'épaisseur, et - il est composé de briques et de pierres, pilées et liées avec de la chaux. Le bleu domine dans les ornemens, - ou les guillochages qui forment l'encadrement. Les cartouches qui sont ornés de vases et d'animaux - sont traités sur des fonds blancs. Les flgures et les animaux sont aussi-bien coloriés que les pierres ont - pu le permettre. L'ouvrage même produit assez d'effet, c'est-à-dire qu'il indique du relief. Le morceau 
* étoit très-bien conservé, lorsqu'il a été découvert, mais la pluye l'a dégradé, et les habitants en ont "détaché différentes parties et l'ont enfin entièrement détruit. "

Cr. Adrien Blanchet, Inventaire des mosaiques de la Gaule, II, p. 107, n. 1156 ; ibid., n. 1154, $1155,1157,1158,1159,1160,1161,1162$ : autres mosaïques trouvées à Bavai.

(4) J.-B. Lambiez, ouv. cit., p. 237 : le a trépied... consiste en trois montans de bronze de deux pieds et demi de haut, cancellé en sautoir et surmonté de trois têtes de bacchantes *.

(5) J.-B. Lambiez, ibid. : " ... figure du Panthère, d'une conservation parfaite... La partie courbe ... est décorée d'un vase d'argent et de petites vignes. "

(6) J.-B. Lambiez, ibid. : "... une des trois têtes des bacchantes, surmontée d'une aigrette en or, et dont la coëffure est décorée de feuilles de petites vignes et de grappes de raisin... .

(7) E. Desjardins, dans Mémoires de la Société d'agriculture de sciences et d'arts séant à Douai, t. XI, 1873, p. 104 : «Dimensions : hauteur, $1 \mathrm{~m} .28$; largeur, $0 \mathrm{~m} .63$; épaisseur $0 \mathrm{~m} .45 . .$. Matière : pierre noire de Bavai. Provenance : Bavai ; collection du cure Carlier, découvert en 1777. Devant cette pierre était une grande urne posée sur un socle cubique. Un dessin exécuté dans l'inventaire du curé (manuscrit de la bibl. de Douai, n. 1086), nous a conservé cette disposition et nous l'avons reproduite dans notre planche. On a trouvé dans cette urne des ossements, trois lacrymatoires en verre, deux lampes et une monnaie d'Hadrien. Ce monument est aujourd'hui au musée de Douai. "

(8) Cf. M. M. HÉnault, Pro Nervia, t. V, pp. 6 sq.

(9) Comme les Brariati, Privatus est un fabricant de pelves. Le bord de pelve ici reproduit fut trouvé le 26 février 1940, fouilles Biévelet. Cl. P. Darche, Pro Nervia, t. I, pp. 61 ss., ou il lit : Biruta ou Brivat, et M. M. Hénault et P. Darche, ibid., t. VI, p. 17.

(10) Sur l'industrie du bronze à Bavai, cf. M. M. Hénacle, Pro Nervia, t. I, pp. 31 sq.

(11) Les 90 piles de carreaux rouges qui portaient la suspensura avaient été détruites. Mais on aperçoit, en file, les traces des implantations de ces piles sur l'aire bétonnée. Les restes du mur qui recoupe cette aire témoignent qu'après avoir cessé d'être un hypocauste, cette construction reçut une nouvelle destination. Des tragments de mosaïque de couleur bleue, blanche, rouge, ont été découverts dans les remblais. 